\title{
Phraseodidaktik an der Schnittstelle zur Grammatik- und Wortschatzarbeit im DaF/DaZ- und im sprachsensiblen (Fach-) \\ Unterricht am Beispiel von festen nehmen-Konstruktionen. Annäherung an eine pragmasemantische Konstruktionsdidaktik
}

\author{
László Ránics (Braunschweig)
}

\begin{abstract}
In this paper, I investigate if phraseodidactic approaches can be transferred to grammar and vocabulary training in foreign language lessons to the same extent. Taking a usage-based approaches to foreign and second language didactics, it is shown that phraseologisms can be subjected to structural semantic analyses in foreign language lessons for learners to develop (i) phraseological language awareness and (ii) adequate phraseological language skills. On the basis of the analyses and the recommendations for basic and advanced phraseological vocabulary, a new approach of pragmatic-semantic construction didactics is introduced.
\end{abstract}

\section{Einleitung und theoretische Vorüberlegungen}

Dank der phraseologischen Forschungserkenntnisse der letzten Jahre und Jahrzehnte in Linguistik und Sprachdidaktik ist die Relevanz der systematischen Vermittlung, Aneignung sowie Anwendung von Phraseologismen im v. a. gesteuerten Fremd- und Zweitspracherwerb u. a. durch Ansätze der Phraseodidaktik mittlerweile unumstritten (cf. z. B. Burger 2015; Dobrovol'skij/Piirainen 2009; Ettinger 2019; Hallsteindóttir/Sajánková/Quasthoff 2006; Heringer 2020; Kühn 1992, 1994; Lapinskas 2013; Lüger 2019). Die Phraseodidaktik hat zum einen die Entwicklung einer phraseologischen Sprachbewusstheit, zum anderen die Ausbildung adäquater phraseologischer Sprachkompetenzen zum Ziel. Die didaktisch-methodischen Schritte des Erkennens, des Verstehens, des Einübens und des Anwendens von Phrasemen rücken somit im Fremd- und Zweitsprachunterricht in den Vordergrund (cf. z. B. Lüger 1997; LorenzBourjot/Lüger 2001; Reder 2015). Als Ausgangspunkt muss festgehalten werden, dass die Grenzen der phraseologischen Kategorien fließend zu betrachten sind (cf. Heringer 2020: 1418; Reder 2015: 75). Zweitens herrscht unter Didaktiker:innen weitestgehend Uneinigkeit darüber, welche Phraseologismen zu welchem Zweck (u. a. zur Erweiterung der linguistischen, soziolinguistischen und pragmatischen Kompetenzen) nach den Niveaustufen des Gemeinsamen europäischen Referenzrahmens für Sprachen (GeR) im Fremdsprachenunterricht (FSU) ${ }^{1}$

\footnotetext{
${ }^{1}$ Die Abkürzung FSU für Fremdsprachenunterricht wird im Beitrag als Synonym für den DaF/DaZ-Unterricht verstanden und verwendet. In Anlehnung an Ettinger (2011: 233) wird Sprachunterricht unter
} 
$\mathrm{zu}$ vermitteln sind. Korpus- und phraseologismusorientierte sowie probandenorientierte empirische phraseologische Untersuchungen sind nach Juska-Bacher (2006: 92-94) sowohl für lexikalische Beiträge als auch für phraseologische Minima und Optima im FSU von großer Relevanz. Um letztere begründet bestimmen zu können, betonen Chrissou/Makos (2018: 21) u. a. in Anlehnung an Hessky (2007) neben den quantitativen (Vorkommenshäufigkeit) und qualitativen (Geläufigkeit) Auswahlkriterien den „hohen kommunikativen Wert“ als ein weiteres Kriterium für die Festlegung der Erwerbsrelevanz von Phrasemen. Die Frage nach dem Vermittlungszweck einzelner Phrasemtypen auf unterschiedlichen Niveaustufen des GeR bleibt aufgrund der ,wenig transparenten und distinktiven Beschreibung“ (Chrissou/Makos 2018: 22) von Phrasemen im GeR ${ }^{2}$ sowie in DaF-Lernmaterialien weiterhin offen. Ettinger (2013: 24f.) betont bspw. auf die persönliche Nützlichkeit des Phrasemerwerbs bezogen, dass im Anfängerunterricht frequente Routineformeln für den aktiven Gebrauch im Vordergrund stehen. Kollokationen und idiomatische Syntagmen sind ihm zufolge aufgrund unabdingbarer fundierter Sprachkenntnisse für den Erwerb auf der Sekundarstufe und im universitären Sprachunterricht relevant. Den Erwerb von Sprichwörtern und Parömien empfiehlt Ettinger (ibd.) für Fortgeschrittene, vor allem für die Förderung von passiven/rezeptiven Sprachkompetenzen. Nach Lüger (2019: 69f.) gelten hingegen neben Routineformeln auch Kollokationen in der Vermittlung als vorrangig. Idiomatische Ausdrücke und satzwertige Phraseme sollten nach einer selektiven Auswahl im Vorfeld den passiven, rezeptiven Kompetenzen zugeordnet werden. Augst (2017: 13-16) plädiert bezogen auf Phraseologismen im weiteren Sinne nach Steyer (2004) für usuelle Wortverbindungen (UWV) sowie deren Einflüsse auf die Ansätze der Phraseodidaktik. Daraus lässt sich in Anlehnung an Chrissou/Makos (2018) schlussfolgern, dass für die Lernmaterialerstellung sowohl eine begründete, den Niveaustufen des GeR zugeordnete Auswahl von Phrasemen als auch ihre „Anordnung in einer sinnvollen Lernprogression sowie ihre prozess-, handlungs- und textorientierte didaktische Aufbereitung in Anlehnung an die Ergebnisse der phraseodidaktischen Forschung" (Chrissou/Makos 2018: 31) höchste Priorität haben sollten. Diese Priorisierung ist anhand phraseologischer und phraseodidaktischer Lehrwerkanalysen nachvollziehbar.

\footnotetext{
Phraseodidaktiker:innen als der Unterricht in der Muttersprache sowie als der Unterricht in der Fremdsprache auf allen Altersstufen und in allen Bildungseinrichtungen (Grundschule, Sekundarstufe, Universität und Fachhochschule, Dolmetscher- und Übersetzerausbildung, Fachsprachenkurse, Erwachsenenausbildung usw.) verstanden.

${ }^{2}$ Nach Chrissou/Makos (2018: 20) ,,erfolgt die differenzierte Beschreibung der sukzessiven Entwicklung der phraseologischen Kompetenz von Sprachniveau zu Sprachniveau im Gemeinsamen Europäischen Referenzrahmen für Sprachen und in Profile Deutsch kaum transparent und distinktiv" und die Thematisierung der Erwerbsrelevanz von Phrasemen ist ,unsystematisch und kaum differenziert“ (ibd). Zwar werden im GeR (Europarat 2001: 111f.) zwischen lexikalischen und grammatischen Elementen in der lexikalischen Kompetenz Unterschiede gezogen sowie im Wortschatzspektrum phraseologische Verweise vorgenommen, dennoch bleibt eine Exaktheit des Abgrenzens der Phrasemtyp-Vermittlungsrelevanz auf unterschiedlichen Sprachniveaustufen aus. Diesem differenzierten Abgrenzen wird im Begleitband des GeR (Europarat 2020: 154f.) ebenso wenig Beachtung geschenkt. Es wird lediglich in Bezug auf die Art der Sprache von einem grundlegenden bis hin zu einem breiten, idiomatischen lexikalischen Repertoire ausgegangen. Dies spiegelt sich im z. T. ausgeweiteten Wortschatzspektrum wider, indem bspw. Deskriptoren/Kann-Beschreibungen wie ,das im eigenen Fachgebiet übliche Spektrum an idiomatischen Wendungen verstehen und angemessen verwenden“ (C1), ,die entsprechenden Kollokationen vieler Wörter ziemlich systematisch verwenden“(B2) im Begleitband ergänzt wurden (cf. Europarat 2020: 155).
} 
Targońska (2015) kommt in ihren Untersuchungen zur Thematisierung von Kollokationen in den Lehrwerken Studio d (Funk/Kuhn/Demme 2006; Funk et al. 2009; Niemann 2008, 2010) und Menschen (Braun-Podeschwa/Habersack/Pude 2015; Breitsameter/Glas-Peters/Pude 2013, 2014; Habersack/Pude/Specht 2013) u. a. zum Schluss, dass Kollokationen in den Lehrwerken der Niveaustufen A2 und B1 des GeR zwar auftreten, diese aber weder explizit thematisiert noch gezielt geübt werden. Die phraseologischen und phraseodidaktischen Analysen von Jazbec/Enčeva (2012) der schriftlichen und mündlichen Texte in den Lehrwerken Aspekte 2 (Koithan et al. 2008a-c), em Hauptkurs (Perlmann-Balme et al. 2010a-d), Deutsch mit Grips 2 (Szablyár et al. 2006a-c) der Niveaustufe B2 des GeR ergeben, dass Phraseologismen i. d. R. von den Lehrwerkautor:innen spontan ausgewählt erscheinen, unterrepräsentiert sind und keiner expliziten didaktischen Aufarbeitung unterzogen werden. Ein ähnliches Bild zeichnet sich in der Untersuchung des phraseologischen Inventars der Lehrwerkreihen Aspekte neu (Koithan et al. 2014a-b; 2015a-b; 2016a-b) und Studio [21] (Funk et al. 2013; 2015a-b) zwischen den GeR Niveaustufen A1 und C1 ab (cf. Ránics 2020: 150-153). Bspw. beschränkt sich Studio [21] (Funk et al. 2013; 2015a-b) vorwiegend auf die Vermittlung von kommunikativen Formeln, Kollokationen sowie Nomen-Verb-Verbindungen. Präpositionale und komparative Phraseologismen gewinnen erst ab der Niveaustufe A2 an Bedeutung. Im Gegensatz dazu ist in der Gewichtung der Vermittlungsrelevanz einzelner Phrasemtypen in den Lehrwerkreihen Aspekte neu (Koithan et al. 2014a-b; 2015a-b; 2016a-b) der Grad der Idiomatizität als entscheidend zu nennen: Je höher die Niveaustufe, desto mehr Idiome werden vermittelt und eingeübt. Von den o. g. Selektionskriterien zur Auswahl von Phraseologismen in Anlehnung an die Niveaustufen des GeR sowie den empirischen Belegen in Bezug auf das phraseologische Inventar ausgewählter DaF/DaZ-Lehrwerke ausgegangen, kann folgendes optionales Stufenmodell des Phrasemerwerbs im DaF/DaZ-Kontext aufgestellt werden:

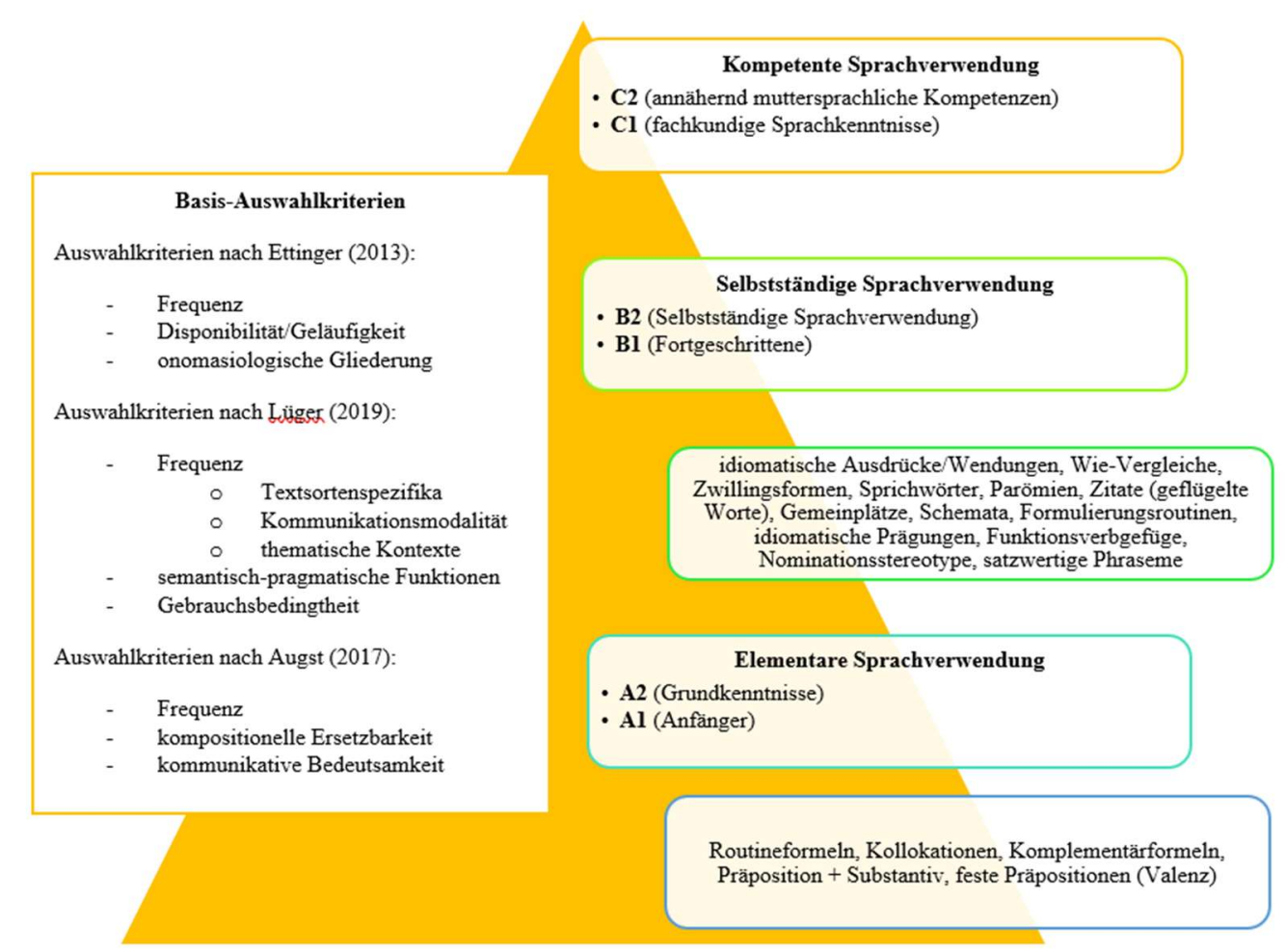

Abbildung 1: Stufenmodell im Phrasemerwerb nach Auswahlkriterien und nach Niveaustufen des GeR 
Das Kriterium der kompositionellen Ersetzbarkeit nach Augst (2017) eröffnet m. E. den Weitblick, um sich mit der Phraseodidaktik an der Schnittstelle zwischen Grammatik- und Wortschatzarbeit im FSU nach den Niveaustufen des GeR und entsprechend den jeweiligen Zielsetzungen der DaF/DaZ-Lernenden (vorrangig das Ablegen einer am GeR orientierten Sprachprüfung) zu befassen. Denn nach der gebrauchsbasierten Spracherwerbstheorie und der Konstruktionsgrammatik (cf. z. B. Stefanowitsch 2011; Tomasello 2006) in Bezug auf die Zweckmäßigkeit der Phrasemschulung zur Erweiterung der linguistischen und somit auch der grammatischen Kompetenzen, ist davon auszugehen, dass Grammatik aus Bedeutungen und Funktionen von mehr oder weniger festen Wortverbindungen entsteht. Konstruktionen werden als ,grundsätzliches grammatisches Ordnungsprinzip“ (Stefanowitsch 2011: 182) aufgefasst (cf. z. B. Behrens 2011: 375-396; Koeppel 2016: 42-44).

In diesem Beitrag werden Phraseologismen in Anlehnung an Stefanowitsch (2011: 181) auch als Konstruktion(en) bezeichnet und aufgefasst. Nach Stumpf (2015: 357) ,gehört also die komplette Phraseologie - sowohl hochfrequente „reguläre“ Kollokationen als auch (scheinbar) grammatisch und/oder semantisch ,irreguläre“ Wortverbindungen, d. h. Wendungen mit formelhaften (Ir-)Regularitäten - zum Gegenstandsbereich der Konstruktionsgrammatik." Des Weiteren wird in Anlehnung an Ziem (2018: 4-5) die Ansicht vertreten, dass sich der Begriff der Konstruktion auf „,sprachliche Größen“ und damit auf mehr oder weniger feste Mehrworteinheiten übertragen lässt. Darüber hinaus ist im Hinblick auf den Spracherwerb nach den Grundprinzipien des gebrauchsbasierten Ansatzes (Intentionen des sprachlichen Handelns, Konstruktions- und Gebrauchsbasiertheit) festzuhalten, „dass Wissen aus dem Gebrauch zu erschließen ist und Spracherwerb somit der Erwerb von Konstruktionen aus dem reellen Sprachgebrauch ist" (Uth 2019: 70-71). Sowohl die Grammatik- als auch die Wortschatzarbeit im FSU lassen sich im Allgemeinen als systematisches Training der Kenntnisse des Vokabulars und der Grammatik einer Sprache sowie als ihre adäquate Anwendung grob beschreiben. Folglich erscheint es aus Sicht der DaF/DaZ-Didaktik und Methodik umso relevanter, bestimmte Konstruktionsschemata - also Form- und Funktionseinheiten der Sprache - auch auf die Ableitbarkeit ihrer lexikalischen und (morpho-)syntaktischen Eigenschaften zu beschreiben. Dadurch kann auch der Phraseodidaktik in Anlehnung an die Ansätze der gebrauchsbasierten Spracherwerbstheorie und der Konstruktionsgrammatik umso mehr Aufmerksamkeit im DaF/DaZ-Unterricht geschenkt werden. Laut Ziem (2018: 5) ist zwar in sprachgebrauchsbedingten Verfestigungsprozessen die kompositionelle Zerlegung komplexer Einheiten in Bestandteile für das angemessene Verstehen und Anwenden nicht notwendig, dennoch sollte diese Möglichkeit im FSU nicht vollkommen ausgeschlossen werden.

Die Entscheidung, mich in diesem Beitrag der Beschreibung von festen nehmen-Konstruktionen zu widmen, erscheint sinnvoll, da Phraseologismen im Allgemeinen - seien sie bspw. Idiome, Kollokationen oder auch Funktionsverbgefüge (FVG) - DaF/DaZ-Lernenden sowie Lehrenden aufgrund von fehlenden überschaubaren Regeln (cf. Kamber 2008: 2) im FSU, aber auch im sprachsensiblen (Fach-)Unterricht sowohl beim Erwerb, Aneignen, Anwenden als auch in der Vermittlung Hürden darstellen können. Andererseits lassen sich unter festen nehmenKonstruktionen unzählige Beispiele für verschiedene Typen von Phraseologismen finden. Die Analysen in Bezug auf ihre Klassifikationsmöglichkeiten, semantischen und (morpho-)syntaktischen Eigenschaften sowie (fremd-)sprachdidaktischen Aspekte erscheinen zielführend, 
zumal im FSU ,,auch unikale Komponenten und morphosyntaktische Besonderheiten als Erkennungshilfen für formelhafte Wendungen bewusst" (Stumpf 2015: 410) gemacht werden sollten.

Somit hat der Aufsatz zum Ziel, die Bedeutung eines phraseologischen Grund- und Aufbauwortschatzes darzustellen sowie konkret am Beispiel von festen nehmen-Konstruktionen aufzuzeigen, wie ein solcher gestaltet und vermittelt werden könnte. Bevor die aus den phraseologischen Mischklassifikationen (Kapitel 3) hervorhegenden semantischen und (morpho-)syntaktischen Analysen von festen (teil-)idiomatischen nehmen-Konstruktionen (Kapitel 3.1) sowie von nehmen-Funktionsverbgefügen (Kapitel 3.2) erfolgen, ist die Frage nach den grundlegenden (fremd-)sprachdidaktischen Relevanzen solcher Konstruktionen zu beantworten (Kapitel 2). Um die Vermittlungsrelevanz anschaulicher darzustellen, wird in Kapitel 2.1 das phraseologische Inventar gängiger DaF/DaZ-Sprachzertifikate extrahiert. Der exemplarische phraseologische Grund- und Aufbauwortschatz nach den Niveaustufen und Kompetenzbereichen des GeR am Beispiel von festen nehmen-Konstruktionen wird in Kapitel 4 vorgestellt. Dieser soll abschließend die Ansätze einer pragmasemantischen Konstruktionsdidaktik für die Ausbildung, Erweiterung und Förderung phraseologischer Kompetenzen (Kapitel 5) sowohl im DaF/DaZ- als auch im sprachsensiblen (Fach-)Unterricht für DaF/DaZ-Lernende (Kapitel 6) greifbarer machen.

\section{Zur (fremd-)sprachdidaktischen Relevanz von festen (nehmen-)Konstruktionen bei Förderung allgemeinsprachlicher Kompetenzen von DaF/DaZ-Lernenden}

Dass Didaktiker:innen seit den 1990er Jahren konstruktionsgrammatischen sowie gebrauchsbasierten spracherwerbstheoretischen Ansätzen und somit der Phraseo-, der Kollokationsdidaktik und dem Chunk-Ansatz im FSU mehr Aufmerksamkeit schenken sollten, ist auch in Anlehnung an Aguado (2008: 57-58) logisch. Sie betont, dass trotz der beachtlichen Leistung von Fremd- und Zweitsprachlernenden Sprachbausteine zu memorieren und diese inhaltlich-situativ sowie formal-strukturell adäquat anzuwenden, deren Wiedergabe und Paraphrasierung im FSU weiterhin höhere Bewertung finde und dadurch die Rolle und Funktion von Phraseologismen im Allgemeinen weiterhin stark unterschätzt werde (cf. auch Koeppel 2016: 43). Die Verwendungsaspekte von Phraseologismen (cf. Donalies 2009: 45-56; Lapinksas 2013: 176-182) lassen sich von ihrem grundsätzlichen Gebrauch, ihrer Funktion zur Versprachlichung über ihre bspw. steuernde und vereinfachende Rolle in der Kommunikation bis hin zur Nomination charakterisieren. Darüber hinaus sind Phraseologismen im weiteren Sinne in Übereinstimmung mit Aguado (2016: 30), ,nützliche Mittel für eine erste Orientierung im unmittelbaren zielsprachlichen Lebensumfeld“ für DaZ-Lernende, damit sie in der Zielsprache angemessen kommunizieren können. Das impliziert auch, dass phraseologische Sprachproduktion den Normalfall kompetenter Sprachkenntnisse darstellt (cf. Aguado 2008: 58). Phraseologische Lernziele rücken im FSU v. a. durch klassematische, grammatische Restriktionen sowie phraseologische Gebrauchsbedingungen im Hinblick auf die (autonome) Entwicklung aktiver und passiver phraseologischer Kompetenzen in den Mittelpunkt (cf. Ettinger 2019: 91-95).

Zwar wird die Bedeutung eines phraseologischen Grund- und Aufbauwortschatzes in DaF/DaZ kontrovers diskutiert, die sprachkompetenzfördernde Rolle sollte aber bspw. aus der Perspektive des ausländischen DaF-Unterrichts u. a. an bilingualen Schulen, an denen viele Schulfächer 
auf Deutsch unterrichtet werden und somit der Zugang zur deutschen Bildungs- und Fachsprache gewährleistet wird, nicht außer Acht gelassen werden. Andererseits ist es aus Sicht des bundesweiten DaZ-Unterrichts u. a. in Anbetracht der obligatorisch abzulegenden Sprachzertifikate und des Übergangs von alltagssprachlichen zu bildungs- und fachsprachlichen Kompetenzen sinnvoll, sich über (fremd- und zweit-)sprachdidaktische und methodische Zugänge zu einem phraseologischen Grund- und Aufbauwortschatz und dadurch zur Entwicklung und Förderung phraseologischer Kompetenzen Gedanken zu machen. Um auf die Relevanz der Schaffung solcher (fremd-)sprachdidaktischen Zugänge im DaF/DaZ- sowie im sprachsensiblen (Fach-)Unterricht empirische Hinweise zu geben, wird im nachstehenden Kapitel das phraseologische Inventar der telc Übungstests für Beruf, Schule zwischen den A2 und C1 Niveaustufen des GeR ohne Anspruch auf Vollständigkeit analysiert.

\subsection{Das phraseologische Inventar allgemein- und berufssprachenspezifischer DaF/DaZ-Übungstests}

In meinen früheren Untersuchungen zum phraseologischen Inventar und zur Überprüfung phraseologischer Kompetenzen in DaF/DaZ-Sprachzertifikaten (cf. Ránics 2020) - wie bspw. im Deutsch-Test für Zuwanderer (DTZ) und in Deutschsprachprüfungen für ein Studium in der Bundesrepublik - hat sich gezeigt, dass in Bezug auf die Erhebung von Phrasemkompetenzen die untersuchten Modellprüfungen keinen einheitlichen Schemata folgen. Weder eine Korrelation zum Kernbereich des phraseologischen Optimums für DaF noch exakt ausformulierte Bewertungskriterien zur Beurteilung des Verstehens und Anwendens von Phrasemen stellen den Regelfall dar. Die Prüfungen erscheinen entweder z. T. entphraseologisiert ${ }^{3}$ oder es werden den Lernenden ohne explizite Phrasemschulung und entsprechende (Vor-)Überprüfung erworbener sowie erweiterter phraseologischer Kompetenzen in der Prüfungsvorbereitung phraseologische Stolpersteine in den Weg gelegt. Hinsichtlich der bereits diskutierten (fremd-)sprachdidaktischen Relevanzen zur Einbettung der Phraseodidaktik in den DaF/DaZ-Unterricht sollen an dieser Stelle die Ergebnisse der Untersuchung zum phraseologischen Inhalt der telc Prüfungen Deutsch A2 und B1 Schule (telc 2014) sowie Deutsch Beruf (telc 2010, 2014, 2015, 2018) zwischen den A2 und C1 Niveaustufen des GeR dargestellt werden. Ziel der Untersuchung war es in erster Linie, ein empirisch begründetes Argument sowohl für den Einsatz eines phraseologischen Grund- und Aufbauwortschatzes als auch für die Nützlichkeit eines darauf aufbauenden bildungs- und berufssprachenspezifischen phraseologischen Wortschatzes, vor allem in Bezug auf den Übergang von alltagssprachlichen zu bildungs-, berufs- und fachsprachlichen Kompetenzen bei DaF/DaZ-Lernenden, zu liefern. Die telc-Prüfungen Deutsch A2 und B1 Schule sowie das Zertifikat Deutsch für Jugendliche (telc 2014) sind allgemeinsprachliche Prüfungen, welche den Nachweis kommunikativer Kompetenzen zeigen sollen, die sowohl im Unterricht gelernt als auch im Alltag erworben wurden. Die Prüfungen orientieren sich bezogen auf Kompetenzstufen, Fertigkeiten im Hören, Lesen, Schreiben und Sprechen sowie hinsichtlich auf Bewertungskriterien am GeR. Aus Platzgründen wird an dieser Stelle auf eine Detailanalyse sowie auf eine Detaildarstellung der extrahierten Phrasemtypen in den einzelnen Prüfungen und

\footnotetext{
${ }^{3}$ Unter entphraseologisierten Sprachprüfungen sind die für Prüfungszwecke bearbeiteten Lese- und Hörverstehenstexte zu verstehen, wobei Ursprungstexten Phraseologismen bspw. entnommen wurden oder Phraseologismen umgeschrieben, paraphrasiert sowie z. T. durch nicht feste Wortverbindungen ersetzt wurden.
} 
ihren Aufgabenstellungen verzichtet und stattdessen ein Gesamtüberblick des phraseologischen Inventars mit Bespielen gegeben.

Phraseologismen sind in jeder Prüfung und in jedem Fertigkeitsbereich der Lese- und Hörverstehenstexte verankert. Zahlenmäßig sind in den Prüfungen Deutsch A2 und B1 Schule jeweils mindestens zwischen 50-60 Phraseologismen extrahierbar. Unter ihnen treten in Anlehnung an Augst (2017) folgende Phrasemtypen auf:

- Zwillingsformen (von Insel zu Insel)

- Wie-Vergleiche (wie im Flug)

- Präposition und Substantiv-Verbindungen (von Beruf)

- feste präpositionale Valenzen (sich freuen auf + Akk., mit jmdm. in Kontakt kommen, in jmds. Gunst)

- Nominationsstereotype (praktische Arbeit, interkulturelles Lernen)

- Funktionsverbgefüge (im Gegensatz zu etw. stehen)

- Kollokationen (Ideen einbringen, Unterricht nehmen, auf Sendung gehen)

- Routineformeln (bis bald, herzlichen Glückwunsch, mit freundlichen Grüßen)

- Formulierungsroutinen (mal überlegen (lassen), zum Schluss, an zweiter Stelle, rund die Hälfte)

- Idiome (zur Welt kommen, jmdn. auf die Nerven gehen, keinen Blatt vor den Mund nehmen, etw. ins Leben rufen, ein alter Hase sein)

Idiome sind demzufolge bereits auf den Niveaustufen A2 und B1 vorzufinden und ähnlich verhält es sich in den Prüfungen Deutsch Beruft zwischen den Niveaustufen A2 und C1, wobei seltener Idiome, dafür aber berufs- und teilweise fachsprachenspezifische Phraseologismen in den Vordergrund rücken:

- Niveaustufe A2: Notdienst unterhalten, etw. in Betrieb nehmen, Wert legen auf + Akk., Kontakte knüpfen mit + Dat.

- Niveaustufe B1: Verständnis haben für etw., betriebsbereiter Computer, Rücksicht nehmen auf + Akk., mittelständisches Unternehmen, innerbetriebliche Weiterbildung, Fehler beheben, ein Teufelskreis sein, nach dem Rechten sehen, in den Ruhestand gehen

- Niveaustufe B2: oberste Priorität haben, zur Verfügung stehen, etw. zum Anlass nehmen, eine/keine (große) Rolle spielen, auf der Strecke bleiben, im Vergleich zu, Status quo, im Durchschnitt, Stellen streichen, im Blickpunkt stehen, Interesse wecken, sich zu Wort melden, mehr oder weniger

- Niveaustufe C1: kräftiger Rückgang, im Mittelfeld, Einfluss haben auf etw., sich großer Beliebtheit erfreuen, Bedeutung zumessen, ausgewogenes Preis-Leistungs-Verhältnis, auf freiwilliger Basis, ein gewichtiges Argument, Zeichen setzen, etw. in Anspruch nehmen, guter Wille, dehnbarer Begriff, alles unter einen Hut bringen können, Frust ablassen, in der Lage sein, in Bezug auf

Diese kurze empirische Analyse zeigt, dass im Grunde genommen alle Phrasemtypen auf allen Niveaustufen des GeR in den allgemein- und berufssprachenspezifischen Sprachprüfungen vertreten sind, auch wenn an dieser Stelle keine Überprüfung von Originaltexten erfolgt ist bzw.

\footnotetext{
${ }^{4}$ Als globales Lernziel wird die berufliche Handlungsfähigkeit in der Fremdsprache Deutsch festgesetzt, wobei die Handlungsfelder mögliche authentische Kommunikationssituationen abbilden und somit keine berufsspezifische Fachsprachenkompetenzen geprüft werden (cf. telc Deutsch B1+ Beruf 2014: 5)
} 
möglich war, um ergänzend für eine Art Entphraseologisierung Belege zu finden. Demzufolge kann davon ausgegangen werden, dass aufgrund der allgegenwärtigen Präsenz von Phraseologismen in jeder Sprache weder im FSU sowie in der abschließenden Überprüfung fremd- und zweitsprachlicher Kompetenzen in Sprachzertifikaten, noch im sprachsensiblen Fachunterricht auf Phraseologismen und demnach auf eine explizite Phrasemschulung verzichtet werden kann. Dies bedeutet, dass neben den grundlegenden Auswahlkriterien wie Frequenz, Geläufigkeit und Gebrauchsbedingtheit für Phraseologismen im FSU - aber auch im sprachsensiblen (Fach-)Unterricht - ergänzend die Auswahlkriterien Verankerung in GeR Sprachzertifikaten, Orientierung an GeR Niveaustufen sowie Fach-, Berufs- und Bildungssprachrelevanzen hinzugefügt werden und im (Sprach-)Unterricht die Ansätze der pragmasemantischen Konstruktionsdidaktik in den Vordergrund rücken sollten. Zunächst soll aber auf die Mischklassifikationen sowie auf die semantischen und (morpho-)syntaktischen Besonderheiten von festen (teil-)idiomatischen nehmen-Konstruktionen und nehmen-Funktionsverbgefügen aufmerksam gemacht werden.

\section{$3 \quad$ Zur Mischklassifikation von festen nehmen-Konstruktionen}

Phraseologismen haben neben ihrer strukturellen und psycholinguistischen Festigkeit ${ }^{5}$ gemein, dass sie mehrgliedrig (polylexikal) sind. Sie bestehen also aus mindestens zwei Worten wie bspw. Stellung nehmen, sind dabei aber nicht länger als ein Satz und somit satz- oder satzgliedwertig wie etw. in Kaufnehmen (cf. Burger 2015: 15). Verglichen mit den angeführten Beispielen für nehmen-Funktionsverbgefüge und Kollokationen ${ }^{6}$ verfügen andere Phrasemtypen wie z. B. Idiome ${ }^{7}$ neben den Eigenschaften der Polylexikalität und Festigkeit über die Eigenschaft der Idiomatizität. Die Bedeutung(en) von Idiomen lassen sich nicht aus der Bedeutung der einzelnen Komponenten ableiten und sie sind zudem - wie das Idiom jn. auf den Arm nehmen im übertragenen Sinne zu verstehen (cf. z. B. Dobrovol'skij/Piirainen 2009: 16). Auf diese Weise kann die kontextualisierte Wortverbindung im Satz Die Mutter nimmt das Kind auf den Arm. wörtlich, aber auch idiomatisch wie im Buchtitel von Hall (2016) Sweet Seventeen: Oder wie mich mein Leben auf den Arm nimmt als ,,(ugs.) jmdn. necken, foppen“ (Duden 2020: 62) verstanden werden.

Im Hinblick auf diese Art von anderen Eigenschaften von Phraseologismen aus struktursemantischer Klassifikationsperspektive und im Gegensatz zu Einzelwörtern sollen an dieser Stelle in Anlehnung an Schlobinski (2005: 15-25) die für den Sprachgebrauch relevanten, jedoch ,vernachlässigten" kommunikativ-stilistischen und pragmatischen Aspekte von Phraseologismen aufgegriffen werden. Feste nehmen-Konstruktionen wie sich etwas nicht nehmen lassen, es

\footnotetext{
${ }^{5}$ Phraseologismen sind ,in einem synchronen Sprachquerschnitt gebräuchlich“ (Burger 2015: 16) und im mentalen Lexikon ähnlich wie ein Wort als Einheit gespeichert, weshalb sie als Ganzes abgerufen und produziert werden (cf. Burger 2015: 11-30).

${ }^{6}$ Kollokationen werden als Phraseme im weiteren Sinne definiert, also als feste, polylexikale, nicht (oder schwach) idiomatische Wortverbindungen (cf. Reder 2015: 74f.; Burger 2015: 38-41). Nach Heringer (2020: 14) werden Kollokationen auch als „Vektoren überfrequent miteinander vorkommender Wörter“ verstanden, die zueinander in semantischer Verbindung stehen.

${ }^{7}$ Nach Dobrovol'skij/Piirainen (2009: 12) stellen Idiome den Kernbereich der Phraseologie dar und sind polylexikal, stabil (Reproduzierbarkeit in nahezu gleicher Form und Bedeutung) sowie idiomatisch nach primärer Lesart und lexikalisierter Bedeutung.
} 
nicht so genau nehmen, wie man's nimmt, Abschied nehmen, von etwas Abstand nehmen, in Betrieb nehmen, etw. zur Kenntnis nehmen usw. werden im Sprachgebrauch situations- und kontextgebunden, oft umgangssprachlich eingesetzt. Sie sind aber (morpho-)syntaktisch komplex $^{8}$, was sowohl im Laufe der (morpho-)syntaktischen Qualifikation (cf. z. B. Niebuhr-Siebert/Baake 2014: 84-100) im Fremd- und Zweitspracherwerb als auch in der Anwendung bei Lernenden zu Schwierigkeiten führen kann. Andererseits stellen Phraseologismen und somit auch die in diesem Beitrag behandelten festen nehmen-Konstruktionen einen wesentlichen Bestandteil der gesprochenen und geschriebenen Sprache dar ${ }^{9}$ und sind demzufolge von großer Relevanz im passiven und aktiven Wortschatzspektrum von DaF/DaZ-Lernenden.

Wie bereits in der Einführung erläutert, sollen nach neueren phraseodidaktischen Forschungserkenntnissen und Ansätzen bspw. Kollokationen, Routineformeln, Formulierungsroutinen und präpositionale Valenzen möglichst bereits zu Beginn des Deutscherwerbsprozesses im FSU vermittelt, angeeignet und angewandt werden (cf. z. B. Augst 2017; Lüger 2019). Die in Kapitel 2.1 dargestellte empirische Analyse zum phraseologischen Inventar ausgewählter Sprachzertifikate zeigt jedoch, dass keine Grenzen zwischen Phrasemtypen auf einzelnen Sprachniveaus gezogen werden, weswegen an dieser Stelle zur Verdeutlichung der Vermittlungsrelevanz die struktursemantische Vielfalt von Phraseologismen am Beispiel von festen nehmen-Konstruktionen beschrieben wird.

Die in diesem Beitrag behandelten festen nehmen-Konstruktionen lassen sich aus linguistischer Sicht teilweise als Mischklassen von Phraseologismen verstehen, weil durch z. B. syntaktische, pragmatische, semantische Kriterien auch neue, spezielle Phrasemklassen entstehen können (cf. Burger 2015: 36). Burger (2015: 61, 171) weist am Beispiel der semantischen Potenzen von Gas geben (als Kollokation: entsprechender Vorgang beim Autofahren vs. als idiomatische Kollokation: ,schnell machen, sich beeilen') auf die Problematik der Grenzziehung zwischen (ir)regulären phraseologischen Verbindungen hin. Kollokationen stellen somit die große Gesamtheit der nicht oder schwach idiomatischen Wortverbindungen dar, welche dadurch zwar für den Sprachgebrauch am bedeutendsten erscheinen, diese aber im Spracherwerb gerade aufgrund ihrer semantischen Potenzen ebenso Schwierigkeiten bereiten können wie Idiome.

$\mathrm{Zu}$ den Kollokationen zählen nicht nur Substantiv-Verb-Kollokationen oder Nomen-Verb-Verbindungen - wie Kollokationen zumeist in DaF/DaZ-Lehrwerken zum Auswendiglernen angeboten werden - , sondern auch Funktionsverbgefüge, die als Komponente ein aus einem Verb nominalisiertes Substantiv (Autosemantikon) und ein semantisch „leeres“ Verb (Synsemantikon) haben und Reihen bilden (können), wie z. B.: Einblick/Einfluss/Rücksicht nehmen.

Helbig/Buscha (2017: 68-94) betonen am Beispiel der Reihenbildung hingegen, dass FVG trotz ihrer Lexikalisierung nicht als Phraseologismen zu identifizieren sind, da das Funktionsverb im FVG eine bestimmte Bedeutung beibehält, während die Bedeutung phraseologischer Einheiten

\footnotetext{
${ }^{8}$ Aus strukturell-linguistischer Perspektive weisen Phraseologismen häufig Irregularitäten in Morphologie und Syntax auf. Manche Phraseologismen verhalten sich bspw. durch die Verbkonjugation oder durch die Kasusverwendung der nominalen Ausdrücke aus (morpho-)syntaktischer Sicht wie beliebige Wortverbindungen, können aber auch externe und interne Valenzen und weitere (morpho-)syntaktische Anomalien aufweisen, wie es bspw. bei Modifikationstypen von Phrasemen der Fall ist (cf. Burger 2015: 12-28, 158-174).

9 „Reales Sprechen und Schreiben sind idiomatisch geprägt, greifbar in den usuellen Wortverbindungen.“ (Augst 2017: 10).
} 
„nur insgesamt fassbar ist“ (Helbig/Buscha 2017: 69). Burger (2015: 57) spricht dementsprechend von einem ,leichten Phraseologisierungsaspekt" am Beispiel von singulären Kollokationen wie eine Frage stellen = fragen und von solchen Wortverbindungen, die kein Verb als Äquivalenzvariante aufweisen können wie im Falle von Geld verdienen. Sowohl Kollokationen als auch ihre Unterkategorie der FVG haben (morpho-)syntaktische, semantische und pragmatische Funktionen und nehmen somit nach Schlobinski (2005: 15-25) als spezifische Konstruktionsschemata eine Zwischenstellung in Lexikalisierungs- und Grammatikalisierungsprozessen ein, können abgeleitet werden, sind z. T. konventionalisiert, aber dennoch kompositionell analysierbar.

\subsection{Zur semantischen und (morpho-)syntaktischen Analyse von festen (teil-)idiomati- schen nehmen-Konstruktionen}

Schlobinski (2005: 15-25) schlägt zur Grammatik- und Freiheitsgradanalyse von Wortverbindungen den Substitutions- und Eliminierungstest vor. Diese Form der Testung ist ebenso bei den Modifikationstypen ${ }^{10}$ von Phrasemen nach Burger (2015: 162) gegeben. Die Substitution bezieht sich auf eine Komponentenersetzung, wodurch die Interpretation des Phrasems im Kontext gesteuert wird, wie im Beispiel „Metzgerin lässt sich ,nicht die Wurst vom Brot nehmen““ (Gießener Allgemeine 05.09.2016). Der Eliminierungstest weist die Verkürzung eines Phrasems auf, wird als Ellipse definiert und lässt sich vor allem in journalistischen Texten sowie in Schlagzeilen wiederfinden (cf. Heringer 2020: 106; Burger 2015: 162-165): bspw. „'Silence Radio': Kein Blatt vor dem Mund“" (Abendzeitung 16.05.2020). Des Weiteren sind auch sog. Phrasemkreuzungen wie im Animalismus ${ }^{11}$ sich wie ein Igel in der Kondomfabrik benehmen (Gerzlich 2021) möglich, abgeleitet aus sich wie ein Elefant im Porzellanladen benehmen. Das Idiom das Leben ist kein Ponyschlecken (Westdeutsche Allgemeine Zeitung 01.04.2016) ist bspw. als „Kreuzung“ von das Leben ist kein Ponyhof und das Leben ist kein Zuckerschlecken zu verstehen. Phrasemkreuzungen können durchaus aus Versprechern und Verschreibern entstehen, die zwar aus struktureller Sicht als Fehler zu klassifizieren sind (cf. Burger 2015: 25), die sich aber dennoch in der Sprache manifestieren können, da sie sowohl geläufig als auch frequent werden und folglich den phraseologischen Wortschatz erweitern. Nicht zuletzt auch aus dem Grund, weil - obwohl Phraseologismen syntaktischen, lexikalischen und grammatischen Restriktionen unterliegen - nachweislich eine Reihe von ,phraseologischen Operationen“ möglich ist, auch wenn in manchen Fällen die Sprachintuition fehlerhafte Äußerungen signalisiert (cf. Burger 2015: 22).

Es soll erstens auf die besondere Rolle von (Ir)Regularitäten auf dem Weg von Valenzschemata zu nehmen-Konstruktionsschemata hingewiesen werden, denn Ágel (2004: 65-66) stellt rückblickend zurecht fest, dass Phraseologismen im Regelfall als valenzsyntaktische sprachliche Zeichen zu identifizieren sind, obwohl ihre Eigenschaft der Mehrgliedrigkeit schon immer

\footnotetext{
${ }^{10}$ Denken wir bspw. in Anlehnung an Burger (2015: 161) an formale und/oder semantische Modifikationstypen von Phraseologismen wie z. B. das Startsignal Auf die Plätzchen, fertig, backt! aus der Sat 1 Fernsehsendung Das große Promibacken (Augsburger Allgemeine 24.03.2021) oder „Die Pizza La Mia Grande ist groß, sogar weit über den Tellerrand hinaus [...] “ in der Pizzawerbung von Dr. Oetker (2021).

${ }^{11}$ In Anlehnung an Heringer (2020: 32-34) werden unter Animalismen Idiome verstanden, in denen Tierbezeichnungen sowie ihre Eigenschaften, Fähigkeiten eine Rolle spielen. Vor allem im Hinblick auf kognitive Modelle zum Verstehen von Animalismen bspw. mithilfe des semantischen Umfelds der Tierbezeichnungen.
} 
Probleme für Valenztheorien darstellt. Folglich sieht Ágel (2004: 69-70) in der strukturellen Valenzrealisierungstheorie die Möglichkeit, Phraseologismen anhand ihrer strukturellen und (morpho-)syntaktischen Variabilität zu beschreiben und schlägt - in Anlehnung an die in der Phraseologieforschung diskutierten internen und externen Valenzen - einen integrativ angelegten Valenzansatz vor, den Stumpf (2015: 219-220) erweitert aufgreift. Dies wird an dieser Stelle anhand des nachstehenden Beispiels verdeutlicht:

\begin{tabular}{|c|c|c|c|}
\hline jemand & nimmt & etwas/jemanden & unter die Lupe \\
\hline externe Valenzstelle & Phrasemkern & externe Valenzstelle & interne Valenzstelle \\
\hline
\end{tabular}

Abbildung 2: Valenzen zu intern fixierten Komponenten

Das angeführte Idiom etw./jmdn. unter die Lupe nehmen verfügt über ein obligatorisches Subjekt jmd. und ein obligatorisches Akkusativobjekt etw./jmdn., welche Leerstellen oder Platzhalter (externe Valenzen) aus dem Bereich der Substantive beliebig ergänzt werden können, wobei weitere quantitative (Valenzwertigkeit), qualitative (Satzgliedfunktion) und selektionale (semantischer Charakter) Aspekte von Bedeutung sind (cf. Burger 2015: 16-24; Stumpf 2015: 219-221), wie bspw. im Falle einer fakultativen adjektivischen Ergänzung in jmd. nimmt etw./jmdn. [scharf] unter die Lupe. Im Gegensatz zur externen Valenz zählen zur internen Valenz die festen Komponenten eines Phrasems. Im Beispielidiom stellt die interne Valenz abhängig vom Idiomkern nehmen das präpositionale Adverbiale unter die Lupe dar, welche zusammen die feste interne Struktur unter die Lupe nehmen bilden. Diese feste innere Struktur ist nicht veränderbar, weswegen in Anlehnung an Stumpf (2015: 220) der Terminus ,interne Valenz“ mit dem Terminus ,intern fixierte Komponente“ ersetzt wird. Hinsichtlich der Beziehung zwischen den intern fixierten Komponenten und der externen Valenzen kann die kasuelle Markierung der Ergänzungen nur unter Berücksichtigung der fixierten Kasusform der internen Ausdrucksstruktur des Idioms gedeutet werden, wobei es aufgrund der Übergänge zwischen veränderbaren und festen Komponenten eines Phrasems Differenzierungsschwierigkeiten zwischen den Valenzstellen gibt (cf. Stumpf 2015: 221). Ágels (2004: 72-79) Stufenmodell der Formvariabilität zum graduellen Verlauf der Valenzstellen stehen Stumpfs (2015: 222-223) Valenz(ir)regularitäten bei verbalen Phrasemen gegenüber. Sowohl Stumpf als auch Burger (2015: 20-21) weisen mit dem Beispiel an jmdm. einen Narren gefressen haben darauf hin, dass es bei einer Reihe von Phraseologismen bei Valenzen des Verbs in phraseologischer und nichtphraseologischer Verwendung Abweichungen geben kann. Das Verb fressen geht mit einem Subjekt und einem Akkusativobjekt einher, verlangt jedoch kein obligatorisches Präpositionalobjekt, weswegen die präpositionale Ergänzung an jmdm. im o. g. Beispiel nicht aus der Valenz des Verbs fressen zu erklären ist. Dies lässt sich auch bei der festen nehmen-Konstruktion ein Beispiel an jmdm./an etw. nehmen erklären. Die präpositionale Dativergänzung an jmdm./an etw. ist nicht aus der Valenz des Verbs nehmen ableitbar und gehört zur internen Phrasemstruktur ein Beispiel nehmen. Betrachtet man ergänzend ein Idiom in Anlehnung an Heringer (2020: 20) als eine Art Schablone von Wortfolgen aus mindestens zwei Wörtern, die unterhalb des Satzes über eine grammatische Struktur mit festen und variablen Besetzungen verfügt, kann das Beispiel etw./jmdn. unter die Lupe nehmen in Struktur und Form folgendermaßen dargestellt werden: 


$\left.\left.\begin{array}{|l|l|l|l|l|}\hline \text { [NP_nom] } & \text { nimmt } & {[\mathrm{NP} \text { _akk] }} & {\left[\begin{array}{l}\text { genauer, genau, kritisch, genauestens, näher, } \\ \text { gründlich, einmal, eingehend, stärker, noch- } \\ \text { mals, intensiv, erneut, kulinarisch, akribisch, } \\ \text { schärfer, streng, dividendentechnisch, genaus- } \\ \text { tens, ausführlich, ausgiebig, gründlicher, peni- } \\ \text { bel, besonders, ebenfalls }\end{array}\right.}\end{array}\right\}\right)$ unter die Lupe

Abbildung 3: Schablonenstruktur mit festen und variablen Besetzungen

Ich stimme Heringer (2020: 19) zu, ,dass in den unterschiedlichen Vorkommensweisen eines Idioms keine klare Grenze zu ziehen ist und dass man vielleicht einen stabilen Kern mit Variation in grammatischen Grenzen und einer freieren Peripherie ansetzen sollte." Beispielsweise ist es bei festen idiomatischen nehmen-Konstruktionen im Passiv möglich, das Semantische vom Syntaktischen zu trennen, indem semantische und (morpho-)syntaktische Eigenschaften auf das semantisch leere idiominterne Akkusativkomplement und auf das idiomexterne Komplement verteilt werden. Die Umformung in das Passiv kann erfolgen, indem eine Nominalphrase in der Komponentenstruktur einer festen idiomatischen nehmen-Konstruktion zum Subjekt promoviert wird: jmdm. den Wind aus den Segeln nehmen wird zu jmdm. wird der Wind aus den Segeln genommen. Das Nomen Wind hat im Idiomkern die relativ selbstständige Bedeutung ,Grund, Voraussetzung für etw.', was die sinnvolle Interpretation des Passivsatzes auch ermöglicht, wie es authentische Kontexte auch belegen: „Auch Boldt gibt zu, interessiert gewesen zu sein, doch ihm wurde schnell der Wind aus den Segeln genommen..." (Hamburger Morgenpost 24.01.2020). Somit sind manche feste idiomatische nehmen-Konstruktionen semantisch zerlegbar, syntaktisch flexibel, wenn die Komponenten(gruppen) im Idiomkern aufgeteilt werden können und die NP einen selbstständigen semantischen Wert hat (cf. Dobrovol'skij/Piirainen 2009: 74-81).

\subsection{Zur semantischen und (morpho-)syntaktischen Analyse von nehmen-Funktions- verbgefügen}

Wie können demzufolge nehmen-Funktionsverbgefüge in Relation zu anderen festen (teil-)idiomatischen nehmen-Konstruktionen in Bezug auf ihre Semantik, Morphosyntax und Valenzstruktur beschrieben werden? Die Entscheidung, mich auch mit nehmen-FVG in diesem Beitrag zu befassen, wurde ähnlich wie bei Kamber (2008: 45-51) nach dem Prinzip getroffen, dass nehmen zu den produktivsten Funktionsverben gehört. Nehmen-FVG (cf. Helbig/Buscha 2017: 68-94) drücken eine Zustands-, Geschehensveränderung oder den Übergang von einem zu einem anderen Zustand aus. Sie können sowohl mit einem Akkusativ (Rache nehmen) als auch in einer Präpositionalgruppe (in Anspruch nehmen) vorkommen und haben eine aktivische Bedeutung. Ein hoher Grad ihrer Festigkeit liegt in ihrer Lexikalisierung begründet. Zumeist sind die nominalen Komponenten Verbalabstrakte (einen Anfang nehmen - anfangen), bei denen eine Paraphrasierung durch das entsprechende Vollverb teilweise möglich ist (ein Bad nehmen - baden). Weder die Substitution des Verbs nehmen durch ein Synonymverb wie bspw. packen, (er)greifen, (er)fassen noch die Anaphorisierung der nominalen Komponenten der lexikalisierten nehmen-FVG (Er nimmt von ihm Abschied. vs. *Er nimmt ihn von ihm.) ist möglich. Gleiches gilt für das Erfragen von Präpositionalgruppen und Akkusativen bei lexikalisierten nehmen-FVG: Er nahm den Apparat in Betrieb kann nicht mit *Wohin nahm er den Apparat? erfragt werden. Des Weiteren ist für nehmen-FVG typisch, dass sie teils mit Nullartikel (Anteil 
nehmen), teils mit dem unbestimmten Artikel (einen schlechten/guten Verlauf nehmen) stehen oder der bestimmte Artikel ist mit der vorangehenden Präposition verschmolzen (zur Kenntnis nehmen).

Bei den nominalen Bestandteilen von lexikalisierten nehmen-FVG besteht auch keine Numerusopposition (in Angriff nehmen vs. *in Angriffe nehmen) und das Anfügen von attributiven Relativsätzen (*Die Zuflucht, die er genommen hat.) sowie von adjektivischen Attributen ( ${ }^{*}$ schnelle Kenntnis nehmen) ist ebenso - i. d. R. im Gegensatz zu nicht lexikalisierten nehmenFVG - nicht möglich. Aus dem Grund weisen auch Helbig/Buscha (2017: 90) auf den hohen Grad der Lexikalisierung und auf die Festigkeit von solchen FVG hin, welche bspw. ohne obligatorische Attribute ungrammatisch sind (eine beachtliche Entwicklung nehmen). Hinsichtlich der Negationsformen lassen sich auch weitere bedeutende Unterschiede aufweisen. Lexikalisierte nehmen-FVG werden einerseits mit nicht (Die Polizei nahm den Täter nicht in Haft.), andererseits im Falle eines Akkusativs mit Nullartikel mit nicht/kein (Er nahm von seinen Eltern nicht/keinen Abschied.) und beim Akkusativ mit unbestimmtem Artikel mit kein (Peter nahm gestern Abend kein Bad.) verneint. Bei einer Negation gilt ergänzend, dass FVG durch Negationswörter in konjunktionalen Nebensätzen nicht zu trennen sind (Der König erwiderte, dass er den Gesandten nicht in Empfang nimmt.) und die Komponenten auch in negierten Aussagesätzen eine Satzklammer bilden: Der Lehrer nimmt den Schüler nicht in Schutz. NehmenFVG sind passivfähig und weisen zumeist - ähnlich wie einige kriegen-Konstruktionen (cf. Schlobinski 2005: 15-25) - eine abstrakte Zustandsveränderung auf (,[...] wurden zu Hilfe genommen, um in Partituren anzuzeigen [...]“ (Wikipedia, s. v. Notation (Musik)).

Weitere syntaktische Bedingungen und Besonderheiten der Valenz von nehmen-FVG drücken sich nicht nur darin aus, dass ihre nominalen Komponenten unabdingbar sind (Die Mutter nimmt das Kind in Schutz. vs. * Die Mutter nimmt das Kind.), sondern auch darin, dass

„beim Übergang eines Vollverbs zu einem FV das betreffende Lexem nicht nur seine ursprüngliche lexikalische Bedeutung verliert, sondern auch die ursprüngliche Valenz; es werden im FVG Valenzverhältnisse konstituiert, die sich quantitativ und qualitativ von den Valenzverhältnissen bei den gleich lautenden Verben unterscheiden“"

(Helbig/Buscha 2017: 91-92).

Die nominalen Komponenten sind die Träger der lexikalischen Bedeutung von FGV, somit hängen die Akkusative und Präpositionalgruppen von ihnen und nicht vom FV ab: Der Lehrer nimmt Einfluss auf die Entwicklung des Schülers. Sie beziehen sich auf das Prädikat, sind eigenständige Satzglieder und umstellbar (cf. Helbig/Buscha 2017: 92): Zu der Aussage nehmen wir Stellung./Wir nehmen zu der Aussage Stellung./Wir nehmen Stellung zu der Aussage.

Bezüglich der semantischen Bedingungen und Leistungen von nehmen-FVG und festen idiomatischen nehmen-Konstruktionen kann anhand der geschilderten (morpho-)syntaktischen und valenzbedingten Analysen festgehalten werden, dass bei beiden Kategorien als Schemata semantische Erweiterungen möglich sind. Bei FVG ist bspw. mithilfe von attributiven Ergänzungen die Schattierung, nähere Charakterisierung möglich (ein böses/gutes Ende nehmen) und gewisse Variationen, Synonymien von Idiomen sind ebenfalls gegeben, wie z. B. jmdn. aufden Arm nehmen/jmdn. auf die Schippe nehmen (cf. Burger 2015: 23). 
In Anbetracht der geschilderten semantischen und (morpho-)syntaktischen Eigenschaften, Anomalien und (Ir)Regularitäten von festen nehmen-Konstruktionen ist es davon auszugehen, dass Phraseme im Allgemeinen für Fremd- und Zweitsprachlernende ohne explizite und phraseodidaktisch-methodische Hinweise auf diese und weitere Restriktionen im Erwerbsprozess Lernprobleme darstellen. Aufgrund dessen stellt sich die Frage nach dem sprach- und phraseodidaktischen und somit auch nach dem pragmasemantisch-konstruktionsdidaktischen Mehrwert von festen, nicht-, teil- oder vollidiomatischen (nehmen-) Konstruktionen im DaF/DaZUnterricht.

\section{Phraseologischer Grund- und Aufbauwortschatz am Beispiel von festen nehmen- Konstruktionen}

Den besten Anhaltspunkt für einen phraseologischen Grund- und Aufbauwortschatz bietet der Kernbereich eines phraseologischen Optimums für den DaF-Grundwortschatz (cf. Hallsteindóttir/Sajánková/Quasthoff 2006) mit 143 Phraseologismen. Ettinger (2019) betont, dass auf Grundlage dieses Optimums Lehrwerke entstehen und weiterführende, möglichst auch kontrastive phraseologische und phraseodidaktische Forschungen u. a. auch in Anlehnung an Ansätze des GeR durchgeführt werden sollten. Auf Basis des phraseologischen Optimums für den DaF-Grundwortschatz habe ich in meiner Forschungsarbeit im Rahmen meiner Dissertation die phraseologischen Kompetenzen von ungarischen DaF/DaN-Lernenden sowie von DaZ-Lernenden in Deutschland miteinander verglichen. Die Ergebnisse dieser Vergleiche (cf. Ránics 2020: 107-119) und das phraseologische Inventar ausgewählter Lehrwerke sowie Sprachprüfungen wurden zum o. g. phraseologischen Kernbereich in Relation gesetzt. Aus diesen Ergebnissen resultiert der Vorschlag, zu den 2400 lexikalischen Einheiten des B1 Grundwortschatzes einen phraseologischen Grundwortschatz aufzubauen. Im Ausgangskorpus werden zu den 2400 Einzelwörtern die zugehörigen Phraseologismen extrahiert und ihre Frequenz in online-Korpora überprüft. Eine differenzierte Aufteilung nach bspw. den Niveaustufen des GeR ermöglicht eine Komponentenüberprüfung in Profile Deutsch (Glaboniat et al. 2017) ${ }^{12}$. Dadurch kann erstens überprüft und zweitens festgelegt werden, welche Komponenten eines Phrasems und im Anschluss welches Phrasem zur welchen Niveaustufe des GeR und zu welchem Kompetenzbereich (rezeptiv oder produktiv) zugeordnet werden kann (cf. z. B. Jesenšek 2006: 140-142; Ránics 2020: 198-202, 228-231).

In Bezug auf die in diesem Beitrag behandelten festen nehmen-Konstruktionen bedeutet dies, ohne eine Frequenzanalyse durchzuführen, Folgendes: Die Verbkomponente nehmen gehört zum Wortschatz des Goethe-Zertifikats B1 (cf. Glaboniat et al. 2016) und ist nach Profile Deutsch auf der Niveaustufe A1 des GeR sowohl dem rezeptiven als auch dem produktiven Kompetenzbereich zugeordnet. Hallsteindóttir/Sajánková/Quasthoff (2006: 133-136) verzeichnen unter den 143 Phraseologismen im Kernbereich des phraseologischen Optimums für DaF drei feste nehmen-Konstruktionen, nämlich etw. (selbst) in die Hand nehmen, etw./jmdn. (mit) in Kauf nehmen, jmdn. beim Wort nehmen. Helbig/Buscha (2017: 79) führen 28 nehmen-

\footnotetext{
12 Profile Deutsch (Glaboniat et al. 2017) ist ein Begleitbuch mit ausführlichen Informationen für Akteure der $\mathrm{DaF} / \mathrm{DaZ}$-Fachbereiche zu GeR Kann- und Kompetenzbeschreibungen, verbunden mit Lernzielbestimmungen und es dient als Hilfe bei der Lehrplanentwicklung, in der inhaltlichen Gestaltung von Lehrbüchern und Prüfungen sowie bei der Planung und Durchführung von Deutschkursen.
} 
FVG in ihrer Liste der Funktionsverben an und Duden (2020 s. v. nehmen) vermerkt mit der Komponente nehmen insgesamt 163 phraseologische Einträge (die o. g. nehmen-Konstruktionen größtenteils inbegriffen). Auf der Grundlage, dass der Duden (2020: 18) „die gebräuchlichsten festen Wendungen der deutschen Gegenwartssprache" dokumentiert, wird an dieser Stelle auf eine Frequenz- und Geläufigkeitsanalyse verzichtet und in Anlehnung an das beschriebene Vorgehen zur Festlegung eines phraseologischen Grundwortschatzes der Versuch unternommen, am Beispiel der extrahierten festen nehmen-Konstruktionen ein differenziertes Optimum aufzustellen.

Berücksichtigt werden dabei die im Beitrag beschriebenen struktursemantischen Kategorien von Phraseologismen und die Relation zu den GeR Kompetenzbereichen. Der phraseologische Grund- und Aufbauwortschatz mit 173 Einträgen zu festen nehmen-Konstruktionen ist der im Anhang stehenden Tabelle zu entnehmen. Der Wortschatz ist zur Kernkomponente nehmen nach Phrasemtypen zu den Nomenkomponenten alphabetisch sortiert sowie den Niveaustufen des GeR und den rezeptiven/produktiven Kompetenzbereichen zugeordnet. Die Aufteilung in einen Grund- und Aufbauwortschatz folgt den Kriterien, zu welcher Niveaustufe des GeR und zu welchem Kompetenzbereich die einzelnen Komponenten wie bspw. Auto- und Synsemantika bei Idiomen oder die Basis und der Kollokator bei Kollokationen gehören. Somit werden 45 feste nehmen-Konstruktionen zum phraseologischen Grundwortschatz (ab Rezeptivniveau A1 - bis Produktivniveau B1) gezählt, da ihre bedeutungstragenden Komponenten zum B1 Grundwortschatz gehören. Bei der Zuordnung zum phraseologischen Aufbauwortschatz der festen nehmen-Konstruktionen (ab Rezeptivniveau B1 - Produktivniveau zwischen B2-C2) spielen der Grad der Idiomatizität, die (morpho-)syntaktische Komplexität sowie in Einzelfällen Unikate eine Rolle. Weitere Beachtung findet die Auffassung von Augst (2017), dass ,unauffälligere" feste Konstruktionen im Fremd- und Zweitspracherwerb den Vorrang vor idiomatischen festen Mehrworteinheiten haben.

\section{Didaktisch-methodische Schritte der pragmasemantischen Konstruktionsdidaktik}

Die Ansätze der pragmasemantischen Konstruktionsdidaktik lassen sich darin begründen, dass bei Fremd- und Zweitsprachlernenden m. E. erst dann messbare Fortschritte hinsichtlich adäquater Fremd- und Zweitsprachenkompetenzen erkennbar gemacht werden können, wenn eine phraseologische Sprachbewusstheit im FSU aktiviert und entwickelt worden ist. Dabei spielen nicht nur die phraseologischen Kompetenzen in der L1 eine wesentliche Rolle - die in Anlehnung an die Überlegungen der Mehrsprachigkeitsdidaktik im FSU immer wieder aufzugreifen sind - sondern auch die Wege der Überführung in die fremd- und zweitsprachlichen phraseologischen Kompetenzen.

Weil phraseologisches Wissen und Können als Normalfall der Sprachverwendung anzusehen sind, ist es im Hinblick auf die Entwicklung linguistischer, soziolinguistischer und pragmatischer Kompetenzen im FSU unabdingbar, Phraseologismen in den Sprachunterricht und somit auch in den sprachsensiblen (Fach-)Unterricht einzubetten. Aus sprachpädagogischer Sicht erscheint es somit als irrelevant, ob man als „Verfechter“ der Konstruktionsgrammatik, der Phraseo- und der Kollokationsdidaktik oder des Chunk-Ansatzes und weiteren gebrauchsbasierten Ansätzen im Unterricht auftritt. Es ist viel mehr von Bedeutung, dass an erster Stelle die Bewusstmachung der sprachpädagogischen phraseologischen Kompetenzen seitens der 
Lehrenden erfolgt, um sich weitere didaktisch-methodische Schritte für den Unterricht zu überlegen. Die didaktisch-methodischen Herangehensweisen sieht die pragmasemantische Konstruktionsdidaktik weder darin ausgeschöpft, dass Phraseologismen als Einheiten vermittelt, angeeignet und angewendet werden, noch darin, dass mehr oder weniger feste Konstruktionen in isolierten Grammatikübungen (morpho-)syntaktischen Analysen unterzogen und solche Strukturen als Fertigteile eingeübt und später angewandt werden.

Vor dem Hintergrund, dass jeder Unterricht Sprachunterricht darstellt, geht es vielmehr darum, dass Form- und Funktionseinheiten als Schemata feste Bestandteile des (Sprach-)Unterrichts bilden, deren struktursemantischen, lexikalischen, (morpho-)syntaktischen, grammatischen und pragmatischen Eigenschaften sowie Funktionen als Lernhilfe erkennbar, verstehbar, analysierbar und anwendbar gemacht werden. Am Beispiel der hier analysierten Sprachprüfungen - die m. E. sowohl aus linguistischer als auch aus sprachdidaktischer Sicht als beachtlich anzusehen sind - ist festzuhalten, dass eine phrasemfreie oder phrasemarme Versprachlichung zwar möglich ist, diese aber nicht tradiert werden kann und vor allem dann nicht sollte, wenn es um den Nachweis adäquater Sprachkenntnisse geht. Darf es linguistisch und sprachdidaktisch als gerechtfertigt betrachtet werden, phraseologische Stolpersteine den Lernenden und Prüflingen in den Weg zu legen, wenn keine explizite Phrasemschulung im Unterricht stattfand? Können die ab und an flüchtigen Antworten mancher Lehrenden wie „Das sagt man so im Deutschen“ und „Das musst du so (auswendig) lernen“ tatsächlich als ausreichend und für Lernende als nachvollziehbar betrachtet werden? Wohl kaum, denn Phraseologismen ermöglichen nicht nur einen kreativen Unterricht, sondern eignen sich für weitreichende Analysen in kontextualisierter Grammatik- und Wortschatzarbeit, wenn der Zugang und die Aufarbeitung phraseologischer Materialien in Lehrwerken explizit in den Vordergrund rücken würden.

Aus diesem Grund erscheinen nach Sprachniveaus des GeR zusammengestellte phraseologische Grund- und Aufbauwortschätze sinnvoll, die als Basis für weiterführende fach-, bildungsund/oder berufssprachliche phraseologische Wortschätze dienen können. Solche Wortschätze könnten statt der üblichen Wortlisten und Glossare feste Bestandteile des (Sprach-)Unterrichts bilden. Auf ihrer Grundlage kann eine methodische Aufarbeitung erfolgen, welche den jeweiligen Lernzielen angepasst ist und das autonome Phrasemlernen gleichermaßen ermöglicht. In der pragmasemantischen Konstruktionsdidaktik ist aus methodischer Sicht die Ausdehnung der phraseodidaktischen Schritte - Erkennen, Erlernen, Einüben, Behalten und situationsadäquate Anwendung (cf. z. B. Kühn 1992; Ettinger 2007) - möglich. Hierbei lehne ich mich an Heringers (2020: 129-140) Überlegungen zum Idiomenlernen an und möchte diese den Leser:innen übertragbar auf Phraseologismen im weiteren Sinne ergänzend im Stufenmodell der pragmasemantischen Konstruktionsdidaktik darlegen: 


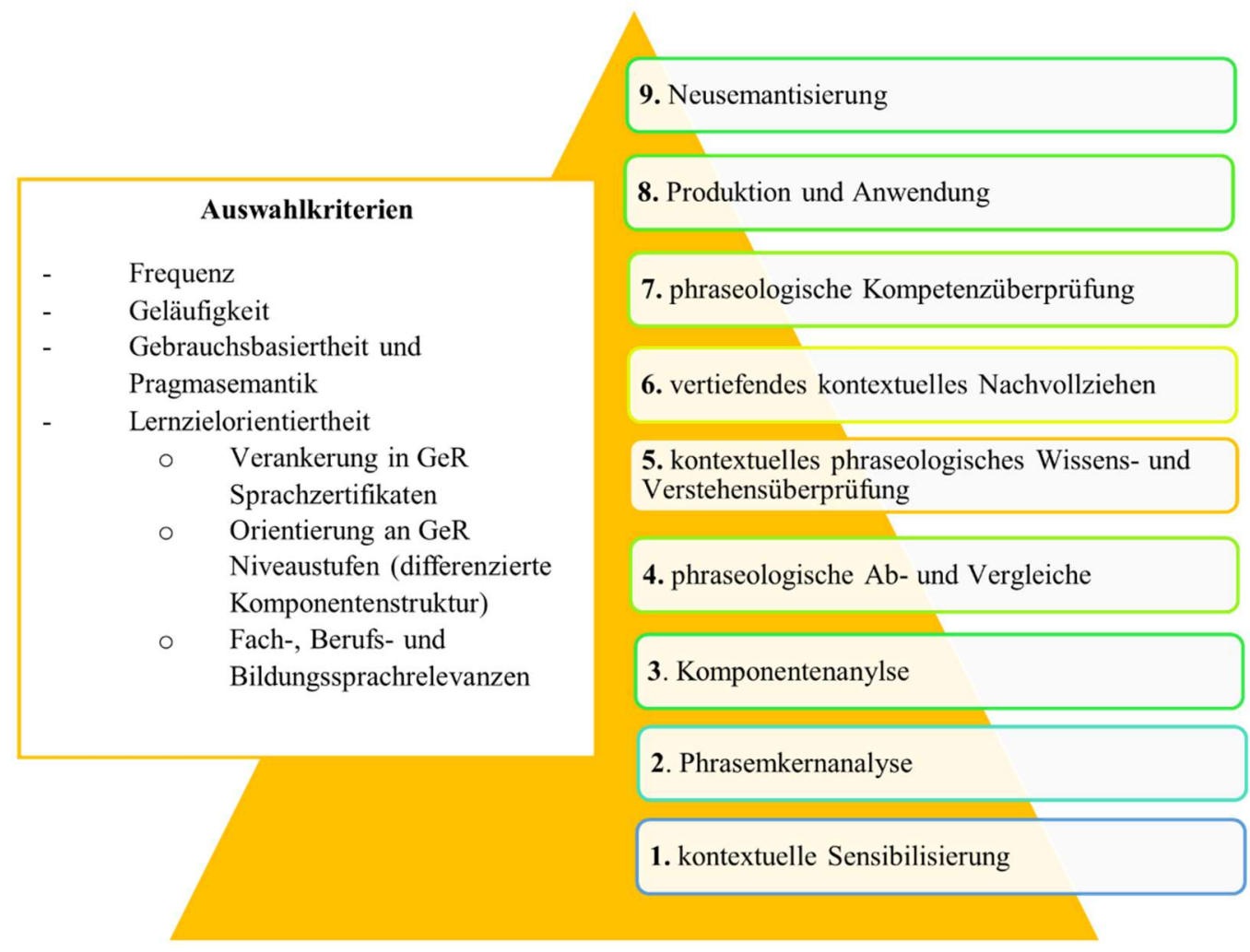

\begin{abstract}
Abbildung 4: Stufenmodell der pragmasemantischen Konstruktionsdidaktik nach differenzierten Auswahlkriterien sowie Operationsmöglichkeiten im FSU ${ }^{13}$
\end{abstract}

Am Beispiel von nehmen-Konstruktionen durch Hervorhebung struktursemantischer Merkmale sollen an dieser Stelle die didaktisch-methodischen Operationsmöglichkeiten in Anlehnung an das Stufenmodell der pragmasemantischen Konstruktionsdidaktik im FSU skizziert werden:

1. Sensibilisierung für kontextualisierte Indizien:

a. bspw. Wortform, grammatische Form, Anführungszeichen im Kontext jmdn. zum Manne nehmen, die Gelegenheit beim Schopfe nehmen, vom Stamme Nimm sein, im Grunde genommen

b. keine wörtliche Deutung möglich jmdn./etw. auf den Haken nehmen

c. Schwierigkeiten in der kontextuellen Dekodierung Peter nimmt den Bus. Er nimmt Rücksicht auf die alten Damen und überlässt ihnen seinen Platz.

d. (fehlende) Verweiswörter im Kontext wie man's nimmt, darauf kannst du Gift nehmen

e. Widerspruch der wörtlichen Deutung ein Herz in die Hand nehmen

f. Unwahrscheinlichkeit der wörtlichen Deutung jmdn. unter die Lupe nehmen

g. Widerspruch der wörtlichen Deutung einzelner Komponenten etw. tierisch ernst nehmen, hart im Nehmen sein

\footnotetext{
${ }^{13}$ Die Auswahlkriterien beziehen sich auf die aktiven/produktiven phraseologischen Kompetenzbereiche. Im Be-
} reich der passiven/rezeptiven phraseologischen Kompetenzbereiche ist keine Grenze nach oben zu setzen. 
2. Herausarbeitung des Phrasemkerns und der Komponenten:

a. Aufmerksamkeit auf bestimmte (grammatische) Strukturen lenken sich ein Beispiel (an jmdm./an etw.) nehmen, jmdn./etw. in Anspruch nehmen, sich die Freiheit nehmen, sich etw. nicht nehmen lassen

b. Charakteristika ausarbeiten, analysieren im Grunde genommen

c. Hervorhebungen der Schablonartigkeit [NP_akk] in Empfang [V_nehmen] [NP_akk] mit ins Grab [V_nehmen]

3. Komponentenanalyse:

a. Assoziationen zum semantischen Umfeld erstellen eine Hürde nehmen vs. *eine Barriere/Barrikade nehmen vs. eine Hürde überspringen

4. Abgleich von Paraphrasen, Synonymen und Antonymen:

a. Hypothesen für geeignete Paraphrasen aufstellen

Unterricht nehmen vs. am Unterricht teilnehmen vs. von jmdm. unterrichtet werden vs. Lehre erteilt bekommen

b. Ersatzprobe mit Synonymen ein Bad nehmen vs. *ein Bad ergreifen, etw. auf die leichte Schulter nehmen vs. *etw. auf die zarte/gewichtslose Schulter nehmen

c. Abgleich von Antonymen Geld verdienen vs. Geld ausgeben $\approx$ Geld zum Fenster hinauswerfen

5. phraseologische Wissens- und Verstehensüberprüfung im Kontext

a. Kontextbefragung, Reihenfolgen erkennen

\section{Märchen 14}

Es war einmal eine Prinzessin, deren Traumprinz einfach nicht heiraten wollte. Und so sprach sie eines Tages zu ihm:

„Ich muss dir jetzt die Pistole auf die Brust setzen. Du bist echt ein alter Halunke, wie dein Vater. Der Apfel fällt nicht weit vom Stamm. Echt, mir steht das Wasser bis zum Hals. Mit dir zusammen zu sein, kommt mir vor, wie Perlen vor die Säue werfen.“

Der Prinz antwortete:

„Du hast doch nicht mehr alle Latten am Zaun. Ich will nicht mehr ständig nach deiner Pfeife tanzen. Immer wieder die alte Leier. Ganz ehrlich: Auf dem Ohr bin ich taub."

Da sagte die Prinzessin plötzlich ganz sanft:

„Du musst ja nicht jedes meiner Worte auf die Goldwaage legen. Komm, jetzt fühl dich nicht auf den Schlips getreten. Schwamm drüber, okay?“

Der Prinz seufzte und sprach:

\footnotetext{
${ }^{14}$ Der „Märchentext“ wurde den „Versprechern“ nach Zerbst (2014: 17) entnommen und in die richtige Form
} überführt. 
„Na gut. Der Drops ist gelutscht. Dann treten wir in den heiligen Bund der Ehe. In der Not frisst der Teufel Fliegen. Du bist zwar wirklich nicht mehr die Jüngste, aber was soll's: Auf alten Schiffen lernt man segeln.“

b. kontrastive phraseologische Vergleiche ziehen (lassen), Äquivalenzanalyse

6. vertiefendes kontextuelles Nachvollziehen

a. Gebrauchsbasiertheit überprüfen (Gebrauchsursprung, Etymologie, Bildungswissen, Übertragbarkeit, Vermutungsfähigkeit)

Jens [...] erzählt, dass sein neuer Mitschüler sehr schüchtern schien: Er habe sich in den Pausen alleine auf eine Bank gesetzt und nicht versucht, sich mit irgendwem zu unterhalten. „Dann musst du ihn mal unter deine Fittiche nehmen“, schlägt Jans Mutter vor. Jan guckt sie verdutzt an. „Worunter soll ich ihn nehmen?“(Kingston 2020)

7. Überprüfung der passiven und aktiven phraseologischen Kompetenzen

a. pragmasemantische Anwendungsaspekte erschließen und einüben

Um pragmasemantische Anwendungsaspekte erschließen, einüben und überprüfen zu können, eignet sich der Einsatz von: Lese- und Schreibtagebüchern, Briefheften sowie weiterführende Übungen im FSU einzubauen wie bspw. Unikalia-Lückentexte, Deutungswege erschließen, formale Auffälligkeiten untersuchen, Phrasemkern- und Komponentenanalysen, Synonym-Proben, Bildstimulus (cf. Heringer 2020: 139-140).

8. Produktion und Anwendung

Zur Produktion und Anwendung von Phraseologismen eignen sich bspw. Übungsformen:

- 10 Phraseme - 1 Text

- Phrasem - Tabu - Spiel

- Phraseme - Zeichnen - Spiel

- Wie viele Wortverbindungen kennst du mit dem Wort X?

- Memix - Das verdrehte Sprichwort-Memo

- Remix - Rekonstruktionsspiel zu kontextualisierten verdrehten Wortverbindungen

9. Neu-Semantisierung im sprachsensiblen (Fach-)Unterricht

mit jmdm. seine Kräfte messen vs. Kräfte messen

Die pragmasemantische Konstruktionsdidaktik sollte sowohl im FSU als auch im sprachsensiblen (Fach-)Unterricht mit ergänzender Semantisierung auf (kontrastive) phraseologische Forschungserkenntnisse aufbauen, die ebenso an konstruktionsgrammatische Ansätze als auch an die Phraseo-, die Kollokationsdidaktik und den Chunk-Ansatz angelegt sind. Sie stehen zueinander in Verbindung und berücksichtigen im hohen Maße pragmatische und semantische Aspekte, die für die Ausbildung, Förderung und Erweiterung adäquater phraseologischer Deund Enkondierungskompetenzen in der Fremd- und Zweitsprache ausschlaggebend sind. Berücksichtigung findet dabei auch der erzielte sowie erleichterte Übergang von allgemeinsprachlichen zu bildungs- und fachsprachlichen Kompetenzen bei DaZ-Lernenden. Demzufolge kann durch die Anwendung pragmasemantisch-konstruktionsdidaktischer Ansätze gewährleistet werden, dass linguistische (Lexik, Grammatik, Semantik, Phonologie, Orthographie), soziolinguistische und pragmatische Kompetenzen den Lernzielen entsprechend für adäquate Fremd- 
und Zweitsprachenkompetenzen ausgebildet, gefördert und stetig erweitert werden. Dabei müssen keine Grenzen zwischen den o. g. Disziplinen aus sprachdidaktisch-methodischer Sicht gezogen werden. Das Gerüst eines „erleichterten Zugangs“ zur Entwicklung phraseologischer Kompetenzen stellt exemplarisch der in Kapitel 4 vorgestellte phraseologische Grund- und Aufbauwortschatz am Beispiel von festen nehmen-Konstruktionen dar.

\section{Ausblick: Zur (fremd-)sprachdidaktischen Relevanz von festen (nehmen-) Kon- struktionen bei Förderung fach- und bildungssprachlicher Kompetenzen von DaF/DaZ-Lernenden}

Die Ausbildung, Förderung und Erweiterung adäquater phraseologischer Kompetenzen in Fremd- und Zweitsprache mithilfe eines phraseologischen Grund- und Aufbauwortschatzes sehe ich auch darin begründet, dass Bildungs- und Fachsprache(n) nicht nur morphologische und syntaktische Besonderheiten, sondern auch fachtypische Sprachstrukturen, komplexe Schemata sowie Muster aufweisen und eine andere Bedeutung mit sich ziehen (können). Dementsprechend teile ich die Meinung von Gätje/Langlotz (2020: 273-307), dass fachliches Lernen zugleich sprachliches Lernen bedeutet und somit die systematische Einbettung fachsprachlicher Fertigteile in die unterrichtliche Kommunikation Vorrang haben sollte, damit fachspezifische musterhafte Ausdrucksformen als Ganzes erlernt werden können. Der von Gätje/Langlotz (2020) empfohlene Aufbau sowie das Stabilisieren einer Musterverfügbarkeit im sprachsensiblen (Fach-)Unterricht kann m. E. bei DaF/DaZ-Lernenden dann zu adäquaten bildungsund fachsprachlichen Kompetenzen führen, wenn entsprechende phraseologische Kompetenzen im Fremd- und/oder Zweitsprachunterricht ausgebildet, gefördert und stetig erweitert wurden.

Weder der Erwerb von Einzelwörtern im FSU noch das Auswendiglernen von Fachwörtern im sprachsensiblen (Fach-)Unterricht fördern im Alleingang angemessene (fach-)sprachliche Kompetenzen. Dies impliziert zugleich, dass sowohl Glossare von Einzelwörtern in DaF/DaZLehrwerken als auch Wortlisten zu fachsprachlichen Termini in Schulbüchern den Lernenden und Lehrenden für erkennbare sprach- und fachübergreifende Kompetenzfortschritte im Sprach- und Fachspracherwerb unzureichend sind. Die Forderung an einen für Phraseologismen im Allgemeinen sensibilisierenden, bewusstmachenden Fremd- und Zweitsprachunterricht ggf. mithilfe eines phraseologischen Grund- und Aufbauwortschatzes ab der A1 Niveaustufe des GeR sowie das grammatische und fachdidaktische Weiterdenken der Konstruktionsgrammatik, das auch Gätje/Langlotz (2020) empfehlen, kann mit dem nachstehenden Beispiel einer Rechenaufgabe verdeutlicht werden:

In einem Rohrsystem befindet sich Wasser unter dem Druck $p=1,3$ bar. Berechne die Kraft $F$, die auf ein Flächenstück $A=3,0 \mathrm{~cm}^{2}$ der Rohrwand wirkt.

(Internetbeleg, freie-referate.de)

Es ist davon auszugehen, dass Lernenden, die für feste Wortverbindungen wie unter Druck sein/stehen oder auch für Konstruktionsschemata wie die Kraft wirkt auf oder eine Kraft ausüben auf im FSU erstens sensibilisiert wurden, zweitens durch phraseologische Bewusstmachung allgemeinsprachliche phraseologische Kompetenzen entwickelt und diese Schritt für Schritt gesteuert und ungesteuert erweitert haben, ein besserer Zugang zu fachspezifischen musterhaften Ausdrucksformen im Fachunterricht gewährleistet wird, auch wenn diese 
fachtypischen Sprachstrukturen im Fachunterricht neu semantisiert werden müssen (cf. Leisen 2011: 11). Diesbezüglich weisen Rincke/Leisen (2015: 11f.) auch darauf hin, dass Wörter wie Druck und Kraft - auch wie im o. g. Beispiel - dadurch zu Fachwörtern werden, weil sie in einer bestimmten sprachlichen Umgebung auftreten. Einige Beispiele für (zumeist präfigierte und attributive) nehmen-Konstruktionen aus der Physik-Fachsprache wären Werte annehmen, Volumen einnehmen, zunehmende Kohäsionskräfte, zunehmende Entropie, Spannung aufnehmen, als konstant annehmen, mit zunehmender Zeit, Raum einnehmen (cf. Grotz 2018). Auch weitere, z. T. idiomatische - im allgemeinsprachlichen Kontext - Beispiele können an dieser Stelle angeführt werden wie in Strömen fließen vs. der Strom fließt, unter Strom stehen, einen Kurzschluss haben, bei jmdm. brennt die Sicherung durch, Kraft(Stoff) tanken, gleichschenkliges Dreieck, wässrige Lösung usw. Rincke/Leisen (2015) plädieren dementsprechend - in Bezug auf die Entscheidung für oder gegen bestimmte Wendungen - für eine gründliche fachliche und fachdidaktische Unterrichtsplanung. Dies gewährleistet, dass Lernende nicht vor vermeidbaren (fach-)sprachlichen Hürden stehen. Gegen solche Hürden könnte in Anlehnung an den phraseologischen Grund- und Aufbauwortschatz im FSU ein Aufbauwortschatz fachspezifischer musterhafter Ausdrucksformen im sprachsensiblen (Fach-)Unterricht Hilfe leisten. In beiden Fällen kämen m. E. auf die jeweiligen Lernziele abgestimmte und kontextualisierte Wortschätze u. a. in Glossaren, Aufgabenstellungen und Übungsformen in Frage. Bis dato sind mir keine linguistischen und (fremd-/zweit-)sprachdidaktischen Forschungen bekannt, die sowohl phraseologische als auch fachsprachenspezifische phraseologische Kompetenzen von DaF/DaZ-Lernenden und/oder von Schüler:innen mit DaZ-Förderbedarf untersuchen, ggf. diese sogar zueinander in Relation setzen. Demzufolge liegen vermutlich bisher weder ein auf die Niveaustufen des GeR abgestimmter phraseologischer Grund- und Aufbauwortschatz noch ein damit in Verbindung gesetzter fachsprachenspezifischer phraseologischer Aufbauwortschatz vor.

\section{$7 \quad$ Fazit}

Auf der Grundlage, dass Phraseologismen im weiteren Sinne sich zwischen den Disziplinen Lexik, Grammatik, Pragmatik und Diskurs (cf. Filatkina 2018: 122) bewegen sowie Forschungsschwerpunkte der Konstruktionsgrammatik und der Phraseologie u. a. in Bezug auf Idiomatizität, kognitive Verfestigung, Musterhaftigkeit und Schematizität, Stabilität und Variabilität auch ähnliche Ansätze und Interessen verfolgen (cf. Ziem 2018: 11-13), sollten diese für die (Fremd-)Sprachdidaktik verknüpfend fruchtbar gemacht werden. Daher lässt sich schlussfolgern, dass der Phrasemschulung im FSU und im sprachsensiblen (Fach-)Unterricht keine Grenzen gesetzt werden sollten, da die Möglichkeiten der Einbettung phraseologischer Lehrund Lernmaterialien und verschiedener Übungsformen sowohl an der Schnittstelle zwischen Grammatik- und Wortschatzarbeit als auch in der fachsprachlichen Neusemantisierung grenzenlos sind. Vielversprechend erscheinen demzufolge die skizzierten methodischen Ansätze der pragmasemantischen Konstruktionsdidaktik, sobald diese auch in der Lehrwerkentwicklung Beachtung finden und den jeweiligen Niveaustufen und Lernzielen des GeR differenziert angepasst werden. 


\section{Literaturverzeichnis}

Aguado, Karin (2008): „Wie beeinflussbar ist die lernersprachliche Entwicklung? Theoretische Überlegungen, empirische Erkenntnisse, didaktische Implikationen“. Fremdsprache Deutsch 38/2008: 53-58.

Aguado, Karin (2016): „Deutsch lernen mit Chunks“. Fremdsprache Deutsch. Zeitschrift für die Praxis des Deutschunterrichts. Deutschunterricht für Lernende mit Migrationshintergrund. Sonderheft/2016: 30-34.

Augst, Gerhard (2017): „Von der idiomatischen Wendung zum Common Sense - Überlegungen zur Phraseodidaktik“. Deutsch als Fremdsprache 1/2017: 10-19.

Ágel, Vilmos (2004): „Phraseologismus als (valenz)syntaktischer Normalfall“. In: Steyer, Kathrin (ed.): Wortverbindungen - mehr oder weniger fest. Berlin, De Gruyter: 65-86.

Behrens, Heike (2011): „Grammatik und Lexikon im Spracherwerb: Konstruktionsprozesse“. In: Engelberg, Stefan/Holler, Anke/Proost, Kristel (eds.): Sprachliches Wissen zwischen Lexikon und Grammatik. Berlin, De Gruyter: 375-396.

Burger, Harald (2015): Phraseologie. Eine Einführung am Beispiel des Deutschen. 5. neu bearbeitete Auflage. Berlin: Schmidt Verlag.

Chrissou, Marios/Makos, Evangelos (2018): „Zum Bekanntheitsgrad erwerbsrelevanter Phraseme des Deutschen. Eine Untersuchung bei Germanistik-Studierenden an der Nationalen Kapodistrias-Universität Athen“. Linguistik Online 89 (2). doi.org/10.13092/lo.89.4272 [24.04.2021]

Dobrovol'skij, Dmitrij/Piirainen, Elisabeth (2009): Zur Theorie der Phraseologie. Kognitive und kulturelle Aspekte. Tübingen: Stauffenburg Verlag.

Donalies, Elke (2009): Basiswissen Deutsche Phraseologie. Tübingen: Francke.

Duden online: duden.de/rechtschreibung [08.06.2021].

Duden (2020): Duden Band 11. Redewendungen. Wörterbuch der deutschen Idiomatik-mehr als 18.000 feste Wendungen, Redensarten und Sprichwörter. 5., vollständig überarbeitete und erweiterte Auflage. Berlin: Dudenverlag.

Ettinger, Stefan (2011): „Einige kritische Fragen zum gegenwärtigen Forschungsstand der Phraseodidaktik“. In: Schäfer, Patrick/Schowalter, Christine (eds.): In mediam linguam. Mediensprache-Redewendungen-Sprachvermittlung. Festschrift für Heinz-Helmut Lüger. Landau, Verlag Empirische Pädagogik: 231-250.

Ettinger, Stefan (2013): „Aktiver Phrasemgebrauch und/oder passive Phrasemkenntnisse im Fremdsprachenunterricht. Einige phraseodidaktische Überlegungen“. In: González Rey, Isabel (ed.): Phraseodidactic Studies on German as a Foreign Language. Phraseodidaktische Studien zu Deutsch als Fremdsprache. Hamburg, Dr. Kovač: 11-30. (=Lingua. Fremdsprachenunterricht in Forschung und Praxis 22).

Ettinger, Stefan (2019): Leistung und Grenzen der Phraseodidaktik. Zehn kritische Fragen zum gegenwärtigen Forschungsstand. PhiN. Philologie im Netz 87/2019: 84-124. web.fu-berlin. $\mathrm{de} / \mathrm{phin} / \mathrm{phin} 87 / \mathrm{p} 87 \mathrm{t6}$.htm [10.09.2020].

Europarat (2001): Gemeinsamer europäischer Referenzrahmen für Sprachen: Lernen, lehren, beurteilen. Berlin etc.: Langenscheidt.

Europarat (2020): Gemeinsamer europäischer Referenzrahmen für Sprachen: Lernen, lehren, beurteilen. Begleitband. Stuttgart: Klett. 
Filatkina, Natalia (2018): „Historische formelhafte Wendungen als Konstruktionen: Möglichkeiten und Grenzen der diachronen Konstruktionsgrammatik“. Linguistik Online, 90/3. doi.org/10.13092/lo.90.4320 [10.11.2020].

Gätje, Olaf/Langlotz, Miriam (2020): „Der Ausbau literater Strukturen in Schulbüchern - Eine Untersuchung von Nominalgruppen in Schulbüchern der Fächer Deutsch und Physik im Vergleich“. In: Langlotz, Miriam (ed.): Grammatikdidaktik - theoretische und empirische Zugänge zu sprachlicher Heterogenität. Baltmannsweiler, Schneider Verlag Hohengehren: 273-308.

Glaboniat, Manuela et al. (2017): Profile Deutsch. Gemeinsamer europäischer Referenzrahmen. Lernzielbestimmungen. Kannbeschreibungen. Kommunikative Mittel. Niveau A1-A2. B1-B2. C1-C2. Stuttgart: Klett.

Glaboniat, Manuela et al. (2016): Goethe-Zertifikat B1. Prüfungsziele, Testbeschreibung. Ismaning: Hueber Grotz, Bernhard (2018): Grundwissen Physik. grund-wissen.de [01.12.2020].

Hallsteindóttir, Erla/Sajánková, Monika/Quasthoff, Uwe (2006): „Phraseologisches Optimum für Deutsch als Fremdsprache. Ein Vorschlag auf der Basis von Frequenz- und Geläufigkeitsuntersuchungen“. Linguistik Online 27/2. doi.org/10.13092/lo.27.746 [10.09.2020].

Helbig, Gerhard/Buscha, Joachim (2017): Deutsche Grammatik. Ein Handbuch für den Ausländerunterricht. Stuttgart: Klett.

Heringer, Hans Jürgen (2020): Idiomatik. Eine Einführung. Brey: mykum Verlag.

Hessky, Regina (2007): „Perspektivenwechsel in der Arbeit mit Phraseologie im DaF-Unterricht“. In: Jesenšek, Vida/Fabčič, Melanija (eds.): Phraseologie kontrastiv und didaktisch. Neue Ansätze in der Fremdsprachenvermittlung. Maribor, Slavistično društvo, Filozofska fakulteta: 9-16.

Jazbec, Saša/Enčeva, Milka (2012): „Aktuelle Lehrwerke für den DaF-Unterricht unter dem Aspekt der Phraseodidaktik“. Porta Linguarum 17: 153-171.

Jesenšek, Vida (2006): „Phraseologie und Fremdsprachenlernen. Zur Problematik einer angemessenen phraseodidaktischen Umsetzung“. Linguistik Online 27/2. doi.org/10.13092 /lo.27.747 [10.09.2020].

Juska-Bacher, Britta (2006): „Phraseologische Befragungen und ihre statistische Auswertung“. Linguistik Online 27/2. doi.org/10.13092/lo.27.745 [24.04.2021].

Kamber, Alain (2008): Funktionsverbgefüge - empirisch. Eine korpusbasierte Untersuchung zu den nominalen Prädikaten des Deutschen. Tübingen: Niemeyer.

Koeppel, Rolf (2016): Deutsch als Fremdsprache - Spracherwerblich reflektierte Unterrichtspraxis. 3., überarbeitete und erweiterte Auflage. Baltmannsweiler: Schneider Verlag Hohengehren.

Kühn, Peter (1992): „Phraseodidaktik. Entwicklungen, Probleme und Überlegungen für den Muttersprachenunterricht und den Unterricht DaF“. Fremdsprachen Lehren und Lernen 21: 169-189.

Kühn, Peter (1994): „Pragmatische Phraseologie: Konsequenzen für die Phraseographie und Phraseodidaktik“. In: Sandig, Barbara (ed.): EUROPHRAS 92 Tendenzen der Phraseologieforschung. Bochum, Brockmeyer: 411-428 (= Studien zur Phraseologie und Parömiologie $1)$. 
Lapinskas, Saulius (2013): Zu ausgewählten theoretischen Problemen der deutschen Phraseologie. Ein Lehrbuch für Studierende der Germanistik. Vilnius: Universität Vilnius.

Leisen, Josef (2011): „Praktische Ansätze schulischer Sprachförderung - Der sprachsensible Fachunterricht.“"hss.de/fileadmin/media/downloads/Berichte/111027_RM_Leisen.pdf [10.09.2020].

Lorenz-Bourjot, Martine/Lüger, Heinz-Helmut (eds.) (2001): Phraseologie und Phraseodidaktik. Wien: Edition Praesens. (= Beiträge zur Fremdsprachenvermittlung, Sonderheft 4).

Lüger, Heinz-Helmut (1997): „Anregungen zur Phraseodidaktik“. In: Beiträge zur Fremdsprachenvermittlung 32: 69-120.

Lüger, Heinz-Helmut (2019): „Phraseologische Forschungsfelder. Impulse, Entwicklungen und Probleme aus germanistischer Sicht“. Beiträge zur Fremdsprachenvermittlung 61: 51-82. heinzhelmutlueger.de/wp-content/uploads/2020/02/06-L\%C3\%BCger-51-82.pdf [10.09.2020].

Niebuhr-Siebert, Sandra/Baake, Heike (2014): Kinder mit Deutsch als Zweitsprache in der Grundschule. Eine Einführung. Stuttgart: Kohlhammer.

Ránics, László (2020): Neue phraseodidaktische Ansätze im DaF/DaZ-Unterricht. Ein deutschungarischer empirischer Vergleich. Hannover: Universität Hannover.

Reder, Anna (2015): Überlegungen zu Tendenzen in der Phraseodidaktik. In: Feld-Knapp, Ilona et al. (eds.): Interaktionen. Festschrift für Katalin Boócz-Barna: 74-89. (= DUfU Deutschunterricht für Ungarn 27).

Rincke, Karsten/Leisen, Josef (2015): „Sprache im Physikunterricht“. In: Kircher, Ernst/Girwidz, Raimund/Häußler, Peter (eds.): Physikdidaktik. Theorie und Praxis. 3. Auflage. Berlin, Springer Spektrum: 635-655.

Schlobinski, Peter (2005): „Pass uff, sonst kriegste heiße Ohren! Zur Schematisierung von kriegen-Konstruktionen“. Der Deutschunterricht 5: 15-23.

Stefanowitsch, Anatol (2011): „Keine Grammatik ohne Konstruktionen: Ein logisch-ökonomisches Argument für die Konstruktionsgrammatik“. In: Engelberg, Stefan/Holler, Anke/Proost, Kristel (eds.): Sprachliches Wissen zwischen Lexikon und Grammatik. Berlin/New York, de Gruyter: 181-210.

Steyer, Kathrin (2004): „Kookkurrenz. Korpusmethodik, linguistisches Modell, lexikografische Perspektiven“. In: Steyer, Kathrin (eds.) (2004): Wortverbindungen - mehr oder weniger fest. Berlin/New York, de Gruyter: 87-116. (= Jahrbuch des Instituts für Deutsche Sprache 2003).

Stumpf, Sören (2015): „Formelhafte (Ir-)Regularitäten. Korpuslinguistische Befunde und sprachtheoretische Überlegungen“. Frankfurt am Main etc.: Lang. (Sprache - System und Tätigkeit 67).

Targońska, Joanna (2015): „Kollokationen in DaF-Lehrwerken - eine empirische Studie zur Erfassung und Widerspiegelung der Kollokativität der Sprache in ausgewählten Lehrwerken“. Zielsprache Deutsch 42/3: 3-27.

Tomasello, Michael (2006): „Konstruktionsgrammatik und früher Erstspracherwerb“. In: Fischer, Kerstin/Stefanowitsch, Anatol (eds.): Konstruktionsgrammatik. Von der Anwendung zur Theorie. Tübingen, Stauffenburg: 19-37.

Uth, Svenja (2019): „Gebrauchsbasierung“. In: Roche, Jörg (ed.): Sprachen lehren. Tübingen, Narr: 69-80. 
Zerbst, Jan (2014): „Der Affe fällt nicht weit vom Stamm“. Das Beste aus dem WortsprichMechteltechtel von ffn. Oldenburg: Lappan.

Ziem, Alexander (2018). „Construction Grammar meets Phraseology: eine Standortbestimmung“. Linguistik Online 90/3. doi.org/10.13092/lo.90.4316 [10.11.2020].

\section{Quellenverzeichnis}

Augsburger Allgemeine (2021): augsburger-allgemeine.de/panorama/Promibacken-Jury-Dasgrosse-Promibacken-2021-Juroren-und-Moderatorin-der-Sendung-am-24-3-21id56614851.html [09.06.2021].

Braasch, Simon/Rebien, Florian (2020): „Keine Chance bei Terodde. Arnesen schnappt dem HSV Bozenik weg!“ In: Hamburger Morgenpost. mopo.de/sport/hsv/keine-chance-beiterodde-arnesen-schnappt-dem-hsv-bozenik-weg--33798340 [16.09.2020].

„'Das Leben ist kein Ponyschlecken“: Mirja Boes in Gladbeck“. In: Westdeutsche Allgemeine Zeitung 2014. waz.de/staedte/gladbeck/das-leben-ist-kein-ponyschlecken-mirja-boes-ingladbeck-id11693902.html [16.09.2020].

Dr. Oetker (2021): Dr Oetker. La Mia Grande. oetker.de/unsere-produkte/la-mia-grande/produktsortiment/ [09.06.2021].

freie-referate.de: freie-referate.de/mathematik/druck-berechnen-6-beispiel-aufgaben-mit-formel [25.10.2020].

Gerzlich, Hans (2020): Bürogeflüster. theater-am-park.de/inhalt/Hans-Gerzlich-Buerogefluester/ [14.09.2020].

Gießener Allgemeine (2016): giessener-allgemeine.de/kreis-giessen/wettenberg-ort848780/ metzgerin-laesst-sich-nicht-wurst-brot-nehmen-12027708.html [14.09.2020]

Hall, Sophie M. (2016): Sweet Seventeen: Oder wie mich mein Leben auf den Arm nimmt. lovelybooks.de/autor/Sophie-M.-Hall/Sweet-Seventeen-Oder-wie-mich-mein-Leben-aufden-Arm-nimmt-1249689550-w [14.06.2021].

Kingston, Laura (2020): „Jemanden unter seine Fittiche nehmen“. In: GEOlino 2020. geo.de/geolino/redewendungen/1733-rtkl-redewendung-jemanden-unter-seine-fittiche-nehmen [17.11.2020].

Stadler, Michael (2020): ,'Silence Radio': Kein Blatt vor dem Mund. Juliana Fanjuls packender Dokumentarfilm ,Silence Radio“ über Mexikos mutigste Radio-Moderatorin“. abendzeitung-muenchen.de/kultur/kino/silence-radio-kein-blatt-vor-dem-mund-art-564045 [14.09.2020].

Wikipedia: de.wikipedia.org/wiki/Notation_(Musik) [15.06.2021].

\section{Lehr- und Lernmittel}

Braun-Podeschwa, Julia/Habersack, Charlotte/Pude, Angela (2015): Menschen B1. Kursbuch. München: Hueber.

Breitsameter, Anna/Glas-Peters, Sabine/Pude, Angela (2013): Menschen A2. Arbeitsbuch. München: Hueber.

Breitsameter, Anna/Glas-Peters, Sabine/Pude, Angela (2014): Menschen B1. Arbeitsbuch. München: Hueber.

Funk, Hermann/Kuhn, Christina/Demme, Silke (2006): Studio d A2. Kurs- und Übungsbuch. Berlin: Cornelsen. 
Funk, Hermann et al. (2009): Studio d B1. Kurs- und Übungsbuch. 3. Auflage. Berlin: Cornelsen.

Funk, Hermann et al. (2013): Studio [21] Grundstufe A1: Gesamtband. Das Deutschbuch. Berlin: Cornelsen.

Funk, Hermann et al. (2015a): Studio [21] Grundstufe A2: Gesamtband. Das Deutschbuch. Berlin: Cornelsen.

Funk, Hermann et al. (2015b): Studio [21] Grundstufe B1: Gesamtband. Das Deutschbuch. Berlin: Cornelsen.

Habersack, Charlotte/Pude, Angela/Specht, Franz (2013): Menschen A2. Kursbuch. München: Hueber.

Koithan, Ute et al. (2008a): Aspekte 2. Lehrwerk. Deutsch als Fremdsprache für die Mittelstufe. Niveaustufe B2. 1. Auflage. Berlin, München, Wien, Zürich, New York: Langenscheidt.

Koithan, Ute et al. (2008b). Aspekte 2. Arbeitsbuch. Deutsch als Fremdsprache für die Mittelstufe. Niveaustufe B2. 1. Auflage. Berlin, München, Wien, Zürich, New York: Langenscheidt.

Koithan, Ute et al. (2008c): Aspekte 2. Lehrerhandreichungen. Deutsch als Fremdsprache für die Mittelstufe. Niveaustufe B2. 1. Auflage. Berlin, München, Wien, Zürich, New York: Langenscheidt.

Koithan, Ute et al. (2014a): Aspekte neu B1 plus. Mittelstufe Deutsch. Lehr- und Arbeitsbuch. Teil 1. München: Klett-Langenscheidt.

Koithan, Ute et al. (2014b): Aspekte neu B1 plus. Mittelstufe Deutsch. Lehr- und Arbeitsbuch. Teil 2. München: Klett-Langenscheidt.

Koithan, Ute et al. (2015a): Aspekte neu. Mittelstufe Deutsch. Lehrbuch. B2. München: KlettLangenscheidt.

Koithan, Ute et al. (2015b): Aspekte neu. Mittelstufe Deutsch. Arbeitsbuch mit Audio-CD. B2. München: Klett-Langenscheidt.

Koithan, Ute et al. (2016a): Aspekte neu. Mittelstufe Deutsch. Lehrbuch. C1. München: KlettLangenscheidt.

Koithan, Ute et al. (2016b): Aspekte neu. Mittelstufe Deutsch. Arbeitsbuch mit Audio-CD. C1. München: Klett-Langenscheidt.

Niemann, Rita Maria (2010): Studio d A2. Sprachtraining. 2. Auflage Berlin: Cornelsen.

Niemann, Rita Maria (2008): Studio d B1. Sprachtraining. Berlin: Cornelsen.

Perlmann-Balme, Michaela et al. (2010a): em Neu Hauptkurs. Lehrwerk: Deutsch als Fremdsprache für die Mittelstufe. Niveaustufe B2. 2. aktualisierte Auflage. Ismaning: Hueber.

Perlmann-Balme, Michaela et al. (2010b): em Neu Hauptkurs. Arbeitsbuch: Deutsch als Fremdsprache für die Mittelstufe. Niveaustufe B2. 2. aktualisierte Auflage. Ismaning: Hueber.

Perlmann-Balme, Michaela et al. (2010c): em Neu Hauptkurs. Lehrerhandreichungen: Deutsch als Fremdsprache für die Mittelstufe. Niveaustufe B2. 2. aktualisierte Auflage. Ismaning: Hueber.

Szablyár, Anna et al. (2006a): Deutsch mit Grips. Lehrwerk: Deutsch als Fremdsprache für die Mittelstufe. Niveaustufe B2. 5. aktualisierte Auflage. Stuttgart-Budapest: Klett.

Szablyár, Anna et al. (2006b): Deutsch mit Grips. Arbeitsbuch: Deutsch als Fremdsprache für die Mittelstufe. Niveaustufe B2. 5. aktualisierte Auflage. Stuttgart-Budapest: Klett. 
Szablyár, Anna et al. (2006c): Deutsch mit Grips, Lehrerhandreichungen: Deutsch als Fremdsprache für die Mittelstufe. Niveaustufe B2. 5. aktualisierte Auflage. Stuttgart-Budapest: Klett.

telc (2010): Übungstest 1. telc Deutsch Beruf. Prüfungsvorbereitung. B1. Frankfurt am Main: telc $\mathrm{GmbH}$.

telc (2014): Handbuch Deutsch Beruf. Frankfurt am Main: telc gGmbH.

telc (2014): Übungstest 1. Deutsch Schule. Prüfungsvorbereitung. A2. Frankfurt am Main: telc $\mathrm{gGmbH}$.

telc (2014): Übungstest 1. Zertifikat Deutsch für Jugendliche. Prüfungsvorbereitung. B1. Frankfurt am Main: telc gGmbH.

telc (2014): Übungstest 1. telc Deutsch Beruf. Prüfungsvorbereitung. C1. Frankfurt am Main: telc $\mathrm{gGmbH}$.

telc (2015): Übungstest 1. telc Deutsch Beruf. Prüfungsvorbereitung. B2. Frankfurt am Main: telc $\mathrm{gGmbH}$.

telc (2015): Übungstest 1. Deutsch-Test für Zuwanderer. Prüfungsvorbereitung. A2-B1.Frankfurt am Main: telc gGmbH.

telc (2018): Übungstest 1. telc Deutsch Beruf. Prüfungsvorbereitung. A2+. Frankfurt am Main: telc $\mathrm{gGmbH}$.

\section{Anhang}

\section{Phraseologischer Grund- und Aufbauwortschatz am Beispiel von festen nehmen-Konstruktionen}

\begin{tabular}{|ll|c|c|c|}
\hline \multicolumn{1}{|c|}{ Phrasem } & $\begin{array}{c}\text { Phra- } \\
\text { semtyp }\end{array}$ & $\begin{array}{c}\text { Rezeptivni- } \\
\text { veau }\end{array}$ & $\begin{array}{c}\text { Produktivni- } \\
\text { veau }\end{array}$ \\
\hline 1. & Woher nehmen und nicht stehlen? & SW & B2 & B2 \\
\hline 2. & sich etwas nicht nehmen lassen & GF & B1 & B2 \\
\hline 3. & etw. auf sich nehmen & GF & B1 & B2 \\
\hline 4. & einen nehmen & K & B1 & B1 \\
\hline 5. & es nicht so genau nehmen & GF & B1 & B1 \\
\hline 6. & wie man's nimmt & GF & A2 & B1 \\
\hline 7. & das Abendmahl auf etwas nehmen & I & B2 & C1 \\
\hline 8. & Abschied nehmen (von) & FVG & A2 & B1 \\
\hline 9. & von etw. Abstand nehmen & FVG & B2 & B2 \\
\hline 10. & etw. auf die leichte Achsel/Schulter & I & B2 & B2 \\
\hline
\end{tabular}

${ }^{15} \mathrm{SW}=$ Sprichwort; GF = Gesprächsfloskel; I = Idiom; FVG = Funktionsverbgefüge; K = Kollokation; FR = Formulierungsroutine; $\mathrm{gW}=$ geflügeltes Wort. 


\begin{tabular}{|c|c|c|c|}
\hline 11. sich in Acht nehmen & $\mathrm{K}$ & $\mathrm{B} 2$ & $\mathrm{~B} 2$ \\
\hline 12. etw. in Acht nehmen & $\mathrm{K}$ & B2 & B2 \\
\hline 13. seinen/einen Anfang nehmen & FVG & A1 & A2 \\
\hline 14. etw. in Angriff nehmen & FVG & $\mathrm{B} 2$ & $\mathrm{~B} 2$ \\
\hline 15. einen (neuen) Anlauf nehmen & $\mathrm{K}$ & B2 & $\mathrm{B} 2$ \\
\hline 16. jmdn./etw. in Anspruch nehmen & FVG & $\mathrm{B} 1$ & $\mathrm{~B} 1$ \\
\hline 17. (keinen) Anstand an etwas nehmen & $\mathrm{K}$ & $\mathrm{B} 2$ & $\mathrm{~B} 2$ \\
\hline 18. an etw. Anstoß nehmen & FVG & B2 & $\mathrm{B} 2$ \\
\hline 19. an etw. Anteil nehmen & FVG & $\mathrm{B} 1$ & $\mathrm{~B} 2$ \\
\hline 20. Anteil an jmdm., etw. nehmen & FVG & B1 & $\mathrm{B} 2$ \\
\hline 21. etw. in Arbeit nehmen & $\mathrm{K}$ & $\mathrm{A} 2$ & $\mathrm{~B} 1$ \\
\hline 22. jmdn. auf den Arm nehmen & $\mathrm{I}$ & $\mathrm{A} 2$ & B1 \\
\hline 23. Aufenthalt nehmen & $\mathrm{K}$ & $\mathrm{A} 2$ & B1 \\
\hline 24. einen Aufschwung nehmen & $\mathrm{K}$ & $\mathrm{B} 2$ & $\mathrm{~B} 2$ \\
\hline 25. Aufstellung nehmen & FVG & $\mathrm{B} 2$ & $\mathrm{~B} 2$ \\
\hline $\begin{array}{l}\text { 26. ein Auge/ein paar Augen voll Schlaf } \\
\text { nehmen }\end{array}$ & I & B1 & $\mathrm{B} 2$ \\
\hline 27. jmdn./etw. in Augenschein nehmen & I & $\mathrm{B} 2$ & B2 \\
\hline 28. von etw. seinen Ausgang nehmen & $\mathrm{K}$ & $\mathrm{B} 1$ & B1 \\
\hline $\begin{array}{l}\text { 29. jmdn./etw. für etw. in Aussicht neh- } \\
\text { men }\end{array}$ & $\mathrm{K}$ & $\mathrm{B} 1$ & $\mathrm{~B} 1$ \\
\hline 30. ein Bad nehmen & FVG & A1 & A1 \\
\hline 31. auf etw. Bedacht nehmen & $\mathrm{K}$ & $\mathrm{B} 2$ & $\mathrm{~B} 2$ \\
\hline $\begin{array}{l}\text { 32. die Beine in die Hand/unter den Arm } \\
\text { nehmen }\end{array}$ & I & $\mathrm{B} 2$ & $\mathrm{~B} 2$ \\
\hline $\begin{array}{l}\text { 33. sich ein Beispiel (an jmdm./an etw.) } \\
\text { nehmen }\end{array}$ & $\mathrm{K}$ & $\mathrm{A} 2$ & B1 \\
\hline 34. etw. in Benutzung nehmen & FVG & $\mathrm{B} 1$ & B1 \\
\hline 35. jmdn./etw. in Beschlag nehmen & $\mathrm{K}$ & $\mathrm{B} 2$ & $\mathrm{~B} 2$ \\
\hline 36. jmdn./etw. unter Beschuss nehmen & $\mathrm{K}$ & $\mathrm{B} 2$ & $\mathrm{~B} 2$ \\
\hline 37. etw. in Besitz nehmen & FVG & $\mathrm{B} 1$ & B1 \\
\hline 38. von etw. Besitz nehmen & $\mathrm{K}$ & B1 & B1 \\
\hline
\end{tabular}




\begin{tabular}{|c|c|c|c|}
\hline 39. in Betrieb nehmen & FVG & $\mathrm{B} 2$ & B2 \\
\hline 40. auf etw. Bezug nehmen & FVG & $\mathrm{B} 1$ & B2 \\
\hline $\begin{array}{l}\text { 41. jmdm. die Binde von den Augen neh- } \\
\text { men }\end{array}$ & $\mathrm{I}$ & $\mathrm{B} 2$ & $\mathrm{C} 1$ \\
\hline 42. kein Blatt vor den Mund nehmen & $\mathrm{I}$ & $\mathrm{B} 2$ & $\mathrm{~B} 2$ \\
\hline 43. einen zur Brust nehmen & $\mathrm{I}$ & $\mathrm{B} 2$ & $\mathrm{C} 1$ \\
\hline 44. (sich) jmdn./etw. zur Brust nehmen & $\mathrm{I}$ & $\mathrm{B} 2$ & $\mathrm{C} 1$ \\
\hline 45. jmdn. buckelkraxen nehmen & $\mathrm{I}$ & $\mathrm{C} 1$ & $\mathrm{C} 2$ \\
\hline $\begin{array}{l}\text { 46. sich nicht die Butter vom Brot nehmen } \\
\text { lassen }\end{array}$ & I & $\mathrm{B} 2$ & $\mathrm{C} 1$ \\
\hline 47. einen auf den Diensteid nehmen & $\mathrm{I}$ & $\mathrm{B} 2$ & $\mathrm{C} 1$ \\
\hline 48. jmdn. in Eid und Pflicht nehmen & $\mathrm{I}$ & $\mathrm{C} 1$ & $\mathrm{C} 1$ \\
\hline 49. (einen) Einblick nehmen (in) & FVG & $\mathrm{B} 2$ & $\mathrm{~B} 2$ \\
\hline 50. Einfluss nehmen (auf) & FVG & $\mathrm{A} 2$ & B1 \\
\hline 51. Einsicht nehmen (in) & FVG & $\mathrm{B} 2$ & $\mathrm{~B} 2$ \\
\hline 52. jmdn./etw. in Empfang nehmen & FVG & A2 & $\mathrm{B} 1$ \\
\hline 53. ein/kein Ende nehmen & $\mathrm{K}$ & A1 & A1 \\
\hline 54. ein böses/kein gutes Ende nehmen & $\mathrm{K}$ & A1 & A2 \\
\hline 55. eine ... Entwicklung nehmen & FVG & A2 & $\mathrm{B} 1$ \\
\hline $\begin{array}{l}\text { 56. Wenn man jmdm. den kleinen Finger } \\
\text { gibt, nimmt er gleich die ganzeHand. }\end{array}$ & SW & B1 & $\mathrm{B} 2$ \\
\hline 57. den/seinen Finkenstrich nehmen & $\mathrm{I}$ & $\mathrm{C} 1$ & $\mathrm{C} 2$ \\
\hline 58. jmdn. unter seine Fittiche nehmen & $\mathrm{I}$ & $\mathrm{B} 2$ & $\mathrm{~B} 2$ \\
\hline 59. seinen Fortgang nehmen & $\mathrm{K}$ & $\mathrm{B} 2$ & B2 \\
\hline 60. jmdn. zur Frau nehmen & $\mathrm{K}$ & A1 & A1 \\
\hline 61. sich die Freiheit nehmen & $\mathrm{K}$ & $\mathrm{A} 2$ & $\mathrm{~B} 1$ \\
\hline 62. geben ist seliger als/denn nehmen & SW & $\mathrm{B} 1$ & $\mathrm{~B} 2$ \\
\hline 63. jmdn. ins Gebet nehmen & $\mathrm{K}$ & $\mathrm{B} 2$ & $\mathrm{~B} 2$ \\
\hline 64. etw. in Gebrauch nehmen & FVG & $\mathrm{B} 2$ & B2 \\
\hline 65. Geld in die Hand nehmen & $\mathrm{I}$ & $\mathrm{B} 1$ & B2 \\
\hline 66. die Gelegenheit beim Schopfe nehmen & $\mathrm{I}$ & $\mathrm{B} 2$ & $\mathrm{C} 1$ \\
\hline 67. jmdn. in Gewahrsam nehmen & FVG & $\mathrm{B} 2$ & $\mathrm{C} 1$ \\
\hline
\end{tabular}




\begin{tabular}{|c|c|c|c|}
\hline 68. darauf kannst du Gift nehmen & I & $\mathrm{B} 2$ & $\mathrm{C} 1$ \\
\hline 69. etw. mit ins Grab nehmen & I & B2 & $\mathrm{C} 1$ \\
\hline $\begin{array}{l}\text { 70. als der Großvater die Großmutter } \\
\text { nahm }\end{array}$ & $\mathrm{gW}$ & B2 & B2 \\
\hline 71. im Grunde genommen & FR & B1 & B1 \\
\hline 72. in Haft nehmen & FVG & $\mathrm{B} 1$ & B2 \\
\hline 73. jmdn./etw. auf den Haken nehmen & I & $\mathrm{C} 1$ & $\mathrm{C} 1$ \\
\hline $\begin{array}{l}\text { 74. jmdn. an/bei den Hammelbeinen neh- } \\
\text { men }\end{array}$ & I & $\mathrm{C} 1$ & $\mathrm{C} 1$ \\
\hline 75. jmdn. an die Hand nehmen & $\mathrm{K}$ & B2 & B2 \\
\hline 76. etw. zur Hand nehmen & $\mathrm{K}$ & $\mathrm{B} 1$ & $\mathrm{~B} 2$ \\
\hline 77. jmdm. etw. aus der Hand nehmen & $\mathrm{K}$ & $\mathrm{A} 2$ & B1 \\
\hline 78. etw. in die/in seine Hand nehmen & $\mathrm{K}$ & A1 & A2 \\
\hline 79. hart im Nehmen sein & I & B1 & $\mathrm{B} 2$ \\
\hline 80. jmdm. das Heft aus der Hand nehmen & $\mathrm{I}$ & $\mathrm{B} 1$ & $\mathrm{~B} 2$ \\
\hline 81. das Heft in die Hand nehmen & $\mathrm{I}$ & $\mathrm{B} 1$ & B2 \\
\hline 82. sich etw. zu Herzen nehmen & I & $\mathrm{A} 2$ & B1 \\
\hline 83. sich ein Herz nehmen & $\mathrm{I}$ & $\mathrm{A} 2$ & B1 \\
\hline 84. ein Herz in die Hand nehmen & I & $\mathrm{B} 1$ & $\mathrm{~B} 2$ \\
\hline 85. zu Hilfe nehmen & FVG & A2 & A2 \\
\hline 86. jmdn. auf die Hörner nehmen & $\mathrm{I}$ & $\mathrm{B} 2$ & $\mathrm{C} 1$ \\
\hline $\begin{array}{l}\text { 87. kein Hund nimmt vor jmdm. ein Stück } \\
\text { Brot weg }\end{array}$ & SW & B2 & $\mathrm{C} 1$ \\
\hline 88. eine Hürde nehmen & $\mathrm{K}$ & $\mathrm{B} 2$ & $\mathrm{~B} 2$ \\
\hline 89. seinen Hut nehmen müssen & $\mathrm{I}$ & $\mathrm{B} 1$ & $\mathrm{~B} 2$ \\
\hline 90. jmdn. an die Kandare nehmen & $\mathrm{I}$ & $\mathrm{C} 1$ & $\mathrm{C} 1$ \\
\hline 91. jmdn. am/beim Kanthaken nehmen & I & $\mathrm{C} 1$ & $\mathrm{C} 1$ \\
\hline 92. etw. auf seine (eigene) Kappe nehmen & $\mathrm{I}$ & $\mathrm{B} 2$ & $\mathrm{~B} 2$ \\
\hline 93. etw. in Kauf nehmen & $\mathrm{K}$ & B1 & B1 \\
\hline 94. etw. zur Kenntnis nehmen & FVG & $\mathrm{A} 2$ & B1 \\
\hline 95. Kenntnis nehmen (von) & FVG & $\mathrm{A} 2$ & B1 \\
\hline 96. etw. in Kommission nehmen & $\mathrm{K}$ & B2 & B2 \\
\hline
\end{tabular}




\begin{tabular}{|c|c|c|c|}
\hline 97. jmdn./etw. aufs Korn nehmen & $\mathrm{I}$ & $\mathrm{B} 2$ & $\mathrm{C} 1$ \\
\hline 98. jmdn. beim Kragen nehmen & $\mathrm{I}$ & $\mathrm{B} 2$ & B2 \\
\hline 99. den Krebsgang nehmen & $\mathrm{I}$ & $\mathrm{C} 1$ & $\mathrm{C} 1$ \\
\hline 100. jmdn. in die Kur nehmen & I & $\mathrm{C} 1$ & $\mathrm{C} 1$ \\
\hline 101. etw. in (die) Kur nehmen & $\mathrm{I}$ & $\mathrm{C} 1$ & $\mathrm{C} 1$ \\
\hline 102. jmdn./etw. mit Kusshand nehmen & I & $\mathrm{B} 2$ & $\mathrm{C} 1$ \\
\hline 103. seinen Lauf nehmen & $\mathrm{K}$ & $\mathrm{B} 1$ & $\mathrm{~B} 2$ \\
\hline $\begin{array}{l}\text { 104. Man muss das Leben eben nehmen, } \\
\text { wie das Leben eben ist. }\end{array}$ & SW & B1 & B1 \\
\hline 105. sich das Leben nehmen & $\mathrm{K}$ & $\mathrm{B} 1$ & $\mathrm{~B} 2$ \\
\hline $\begin{array}{l}\text { 106. es von den Lebenden/Lebendigen neh- } \\
\text { men }\end{array}$ & SW & $\mathrm{B} 2$ & $\mathrm{C} 1$ \\
\hline 107. jmdn. in Lohn und Brot nehmen & $\mathrm{I}$ & $\mathrm{B} 2$ & $\mathrm{C} 1$ \\
\hline $\begin{array}{l}\text { 108. jmdm., einer Sache die Luft zum At- } \\
\text { men nehmen }\end{array}$ & $\mathrm{I}$ & $\mathrm{B} 2$ & B2 \\
\hline $\begin{array}{l}\text { 109. jmdn./etw. (scharf) unter die Lupe } \\
\text { nehmen }\end{array}$ & $\mathrm{I}$ & $\mathrm{B} 2$ & B2 \\
\hline 110. jmdn. in die Mache nehmen & $\mathrm{I}$ & $\mathrm{B} 2$ & $\mathrm{C} 1$ \\
\hline 111. etw. in die Mache nehmen & $\mathrm{I}$ & $\mathrm{B} 2$ & $\mathrm{C} 1$ \\
\hline 112. jmdn. in die Mangel nehmen & $\mathrm{I}$ & $\mathrm{B} 2$ & $\mathrm{C} 1$ \\
\hline 113. jmdn. zum Mann(e) nehmen & $\mathrm{K}$ & A1 & A1 \\
\hline 114. jmdn. Maß nehmen & $\mathrm{I}$ & $\mathrm{B} 2$ & $\mathrm{C} 1$ \\
\hline 115. jmdn. unters Messer nehmen & $\mathrm{I}$ & $\mathrm{B} 2$ & $\mathrm{C} 1$ \\
\hline 116. den Mund voll nehmen & $\mathrm{I}$ & $\mathrm{B} 1$ & $\mathrm{~B} 2$ \\
\hline 117. den Mund zu voll nehmen & $\mathrm{I}$ & $\mathrm{B} 1$ & $\mathrm{~B} 2$ \\
\hline 118. etw. nicht in den Mund nehmen & $\mathrm{I}$ & $\mathrm{B} 1$ & $\mathrm{~B} 1$ \\
\hline 119. etw. für bare Münze nehmen & $\mathrm{I}$ & $\mathrm{B} 2$ & $\mathrm{~B} 2$ \\
\hline 120. hart im Nehmen sein & $\mathrm{I}$ & $\mathrm{B} 1$ & $\mathrm{~B} 2$ \\
\hline 121. Notiz von jmdm./von etw. nehmen & $\mathrm{I}$ & $\mathrm{B} 1$ & $\mathrm{~B} 2$ \\
\hline 122. jmds. Partei/für jmdn. Partei nehmen & $\mathrm{I}$ & $\mathrm{B} 1$ & $\mathrm{~B} 2$ \\
\hline 123. etwas persönlich nehmen & $\mathrm{K}$ & A1 & A2 \\
\hline 124. jmdn. in die Pflicht nehmen & $\mathrm{I}$ & $\mathrm{B} 1$ & $\mathrm{~B} 2$ \\
\hline
\end{tabular}




\begin{tabular}{|c|c|c|c|}
\hline 125. Platz nehmen & $\mathrm{K}$ & A1 & A1 \\
\hline 126. etw. zu Protokoll nehmen & $\mathrm{K}$ & B2 & $\mathrm{B} 2$ \\
\hline 127. Quartier nehmen & $\mathrm{K}$ & $\mathrm{B} 2$ & $\mathrm{~B} 2$ \\
\hline 128. an jmdm. Rache nehmen & FVG & $\mathrm{B} 2$ & $\mathrm{~B} 2$ \\
\hline 129. etw. auf seine Rechnung nehmen & $\mathrm{K}$ & $\mathrm{B} 1$ & $\mathrm{~B} 2$ \\
\hline 130. Reißaus nehmen & $\mathrm{K}$ & $\mathrm{B} 2$ & $\mathrm{C} 1$ \\
\hline 131. jmdn. auf die Rolle nehmen & $\mathrm{I}$ & $\mathrm{B} 2$ & $\mathrm{C} 1$ \\
\hline 132. (auf jmdn./etw.) Rücksicht nehmen & FVG & $\mathrm{B} 1$ & B1 \\
\hline 133. mit jmdm. Rücksprache nehmen & $\mathrm{K}$ & $\mathrm{B} 2$ & $\mathrm{~B} 2$ \\
\hline 134. (an etw.) Schaden nehmen & $\mathrm{K}$ & $\mathrm{B} 1$ & $\mathrm{~B} 2$ \\
\hline 135. jmdn. auf die Schippe nehmen & $\mathrm{I}$ & $\mathrm{B} 2$ & $\mathrm{C} 1$ \\
\hline 136. jmdn. am/beim Schlafittchen nehmen & I & $\mathrm{C} 1$ & $\mathrm{C} 1$ \\
\hline 137. den Schleier nehmen & I & $\mathrm{B} 2$ & $\mathrm{C} 1$ \\
\hline 138. jmdn./etw. ins Schlepptau nehmen & I & B2 & $\mathrm{C} 1$ \\
\hline 139. jmdn. beim Schlips nehmen & I & $\mathrm{B} 2$ & $\mathrm{C} 1$ \\
\hline 140. jmdn. in die Schraube nehmen & I & $\mathrm{C} 1$ & $\mathrm{C} 2$ \\
\hline 141. etw. auf die leichte Schulter nehmen & I & $\mathrm{B} 2$ & $\mathrm{~B} 2$ \\
\hline 142. jmdn. aus der Schusslinie nehmen & I & $\mathrm{B} 2$ & $\mathrm{C} 1$ \\
\hline 143. jmdn. in Schutz nehmen & FVG & B1 & $\mathrm{B} 2$ \\
\hline 144. jmdn. zur Seite nehmen & $\mathrm{K}$ & B1 & B1 \\
\hline $\begin{array}{l}\text { 145. einer Sache die Spitze/den Stachel } \\
\text { nehmen }\end{array}$ & I & $\mathrm{C} 1$ & $\mathrm{C} 1$ \\
\hline 146. vom Stamme Nimm sein & SW & $\mathrm{C} 1$ & $\mathrm{C} 1$ \\
\hline 147. Stellung nehmen (zu) & FVG & $\mathrm{B} 2$ & $\mathrm{~B} 2$ \\
\hline 148. jmdn. in Strafe nehmen & $\mathrm{K}$ & $\mathrm{B} 2$ & $\mathrm{~B} 2$ \\
\hline 149. den Strick nehmen & $\mathrm{I}$ & $\mathrm{B} 2$ & $\mathrm{C} 1$ \\
\hline $\begin{array}{l}\text { 150. Gibt man dem Teufel den kleinen Fin- } \\
\text { ger, so nimmt er die ganze Hand. }\end{array}$ & SW & $\mathrm{B} 2$ & B2 \\
\hline 151. etw. tierisch ernst nehmen & $\mathrm{K}$ & $\mathrm{B} 1$ & $\mathrm{~B} 2$ \\
\hline $\begin{array}{l}\text { 152. jmdm. die Trümpfe aus der Hand neh- } \\
\text { men }\end{array}$ & $\mathrm{I}$ & $\mathrm{B} 2$ & $\mathrm{C} 1$ \\
\hline 153. Unterricht nehmen & $\mathrm{K}$ & $\mathrm{A} 2$ & $\mathrm{~B} 1$ \\
\hline
\end{tabular}




\begin{tabular}{|c|c|c|c|}
\hline 154. jmdn. ins Verhör nehmen & $\mathrm{K}$ & $\mathrm{B} 2$ & B2 \\
\hline $\begin{array}{l}\text { 155. einen guten/schlechten Verlauf neh- } \\
\text { men }\end{array}$ & FVG & $\mathrm{B} 2$ & $\mathrm{~B} 2$ \\
\hline 156. etw. in Verwahrung nehmen & FVG & $\mathrm{B} 2$ & $\mathrm{~B} 2$ \\
\hline 157. jmdn., etw. ins Visier nehmen & $\mathrm{K}$ & $\mathrm{B} 2$ & $\mathrm{~B} 2$ \\
\hline 158. jmdn. nicht für voll nehmen & I & $\mathrm{B} 2$ & B2 \\
\hline 159. mit jmdm., etw. vorlieb nehmen & $\mathrm{K}$ & $\mathrm{B} 2$ & $\mathrm{C} 1$ \\
\hline 160. den Weg zwischen die Beine nehmen & I & $\mathrm{B} 1$ & $\mathrm{~B} 2$ \\
\hline 161. jmdn. beim/am Winkel nehmen & I & $\mathrm{C} 1$ & $\mathrm{C} 1$ \\
\hline 162. den guten Willen für die Tat nehmen & $\mathrm{gW}$ & $\mathrm{B} 2$ & $\mathrm{C} 1$ \\
\hline $\begin{array}{l}\text { 163. jmdm. den Wind aus den Segeln neh- } \\
\text { men }\end{array}$ & I & $\mathrm{B} 2$ & $\mathrm{~B} 2$ \\
\hline 164. jmdn. beim Wort nehmen & $\mathrm{I}$ & $\mathrm{B} 1$ & B1 \\
\hline 165. das Wort nehmen & $\mathrm{K}$ & $\mathrm{A} 2$ & B1 \\
\hline $\begin{array}{l}\text { 166. jmdm. das Wort aus dem Mund(e)/von } \\
\text { der Zunge nehmen }\end{array}$ & $\mathrm{I}$ & B1 & B2 \\
\hline 167. etw. in Zahlung nehmen & $\mathrm{K}$ & $\mathrm{B} 1$ & $\mathrm{~B} 2$ \\
\hline 168. jmdn. in die Zange nehmen & I & $\mathrm{C} 1$ & $\mathrm{C} 1$ \\
\hline 169. Zeit nehmen müssen & $\mathrm{K}$ & A1 & A2 \\
\hline 170. sich Zeit nehmen & $\mathrm{K}$ & A1 & A1 \\
\hline 171. sich Zeit für jmdn./etw. nehmen & $\mathrm{K}$ & A1 & A2 \\
\hline $\begin{array}{l}\text { 172. Wer nicht kommt zur rechten Zeit, der } \\
\text { muss nehmen, was übrigbleibt. }\end{array}$ & SW & B1 & B2 \\
\hline 173. seine Zuflucht zu etw. nehmen & FVG & $\mathrm{B} 2$ & $\mathrm{C} 1$ \\
\hline
\end{tabular}

